\title{
GALAXY MERGERS DRIVE SHOCKS: AN INTEGRAL FIELD STUDY OF GOALS GALAXIES
}

\author{
J. A. Rich ${ }^{1,2}$, L. J. Kewley ${ }^{3}$, AND M. A. Dopita ${ }^{3,4}$ \\ ${ }^{1} \mathrm{IPAC}_{2}$ California Institute of Technology, 1200 E. California Boulevard, Pasadena, CA 91125, USA; jrich@ipac.caltech.edu \\ ${ }^{2}$ Observatories of the Carnegie Institution of Washington, 813 Santa Barbara Street, Pasadena, CA 91101, USA \\ ${ }^{3}$ Research School of Astronomy and Astrophysics, Australian National University, Cotter Road, Weston ACT 2611, Australia \\ ${ }^{4}$ Astronomy Department, Faculty of Science, King Abdulaziz University, P.O. Box 80203, Jeddah, Saudi Arabia \\ Received 2015 July 8; accepted 2015 September 22; published 2015 December 7
}

\begin{abstract}
We present an integral field spectroscopic study of radiative shocks in 27 nearby ultraluminous and luminous infrared galaxies (U/LIRGs) from the Great Observatory All-sky LIRG Survey, a subset of the Revised Bright Galaxy Sample. Our analysis of the resolved spectroscopic data from the Wide Field Spectrograph focuses on determining the detailed properties of the emission-line gas, including a careful treatment of multicomponent emission-line profiles. The resulting information obtained from the spectral fits is used to map the kinematics of the gas, sources of ionizing radiation, and feedback present in each system. The resulting properties are tracked as a function of merger stage. Using emission-line flux ratios and velocity dispersions, we find evidence for widespread, extended shock excitation in many local U/LIRGs. These low-velocity shocks become an increasingly important component of the optical emission lines as a merger progresses. We find that shocks may account for as much as half of the $\mathrm{H} \alpha$ luminosity in the latest-stage mergers in our sample. We discuss some possible implications of our result and consider the presence of active galactic nuclei and their effects on the spectra in our sample.
\end{abstract}

Key words: galaxies: active - galaxies: interactions - galaxies: ISM - galaxies: Seyfert - galaxies: starburst

Supporting material: figure set

\section{INTRODUCTION}

As gas-rich starburst galaxies and massive mergers, ultraluminous and luminous infrared galaxies (U/LIRGs) in the local universe provide valuable insight and context for phenomena that impact galaxy formation and evolution at high redshift. ULIRGs, with total $L_{\mathrm{IR}} / L_{\odot}>10^{12}$, are overwhelmingly represented by systems in the latest stages of a merger between two gas-rich galaxies (Sanders et al. 1988; Veilleux et al. 1995; Murphy et al. 1996; Genzel et al. 1998; Rigopoulou et al. 1999; Scoville et al. 2000; Kewley et al. 2001b; Spoon et al. 2006; Desai et al. 2007). The strong infrared luminosity $\left(L_{\mathrm{IR}}\right)$ in local LIRGs is powered by star formation and in some cases active galactic nuclei (AGNs), both driven by galaxy mergers and interactions. LIRGs $\left(L_{\mathrm{IR}} / L\right.$ $\odot>10^{11}$ ) in the high-redshift universe may commonly be mergers, as supported by observational evidence of disturbed morphologies in submillimeter galaxies (SMGs) and an increase in merger activity with redshift (Blain et al. 2002; Dasyra et al. 2008; Bundy et al. 2009; de Ravel et al. 2009). In the local universe, however, a significant fraction $(\sim 20 \%-$ $40 \%$ ) of LIRGs show little or no evidence of ongoing or past strong interactions (Ishida 2004, J. Howell et al. 2015, in preparation). In order to fully understand the processes that govern star formation and black hole assembly, a clear and complete picture of the evolution of U/LIRGs in the local universe is essential.

Integral field spectroscopy (IFS) furnishes one of the most powerful tools for the study of nearby U/LIRGs. Through its ability to both spatially and spectrally map a galaxy, it provides a wealth of spatially resolved information tracing the kinematics of the gas and stars, variations in the radiation field and sources of ionizing photons, metallicity and chemistry in the interstellar medium (ISM), maps of extinction, and gas flows.
IFS studies in recent years have enabled the discovery of extended shock excitation in several nearby galaxies, notably in U/LIRGs and other massive galaxies. Monreal-Ibero et al. (2006) identified extended LINER-like emission resulting from shocks in a small sample of ULIRGs. Sharp \& Bland-Hawthorn (2010) found widespread shock excitation in the extended emission associated with outflows in a study of several galactic wind-bearing galaxies. A larger IFS study found a significant fraction of tidally induced shock excitation in nearby LIRGs (Monreal-Ibero et al. 2010). Farage et al. (2010) discovered extended shocks caused by gas accreting onto a giant brightest cluster galaxy (BCG). Finally, Rich et al. (2010) found extended shock excitation caused by a galactic wind in the M82-like galaxy NGC 839 and widespread shocks in the latestage LIRG mergers NGC 3256 and IC 1623 (Rich et al. 2011). In Farage et al. (2010) and Rich et al. (2010, 2011), new slow shock models were employed to analyze the shocked gas. In all of the above cases, shock excitation exhibits characteristics of extended LINER-like emission with broadened line profiles.

The shock excitation seen in the LIRGs NGC 839, NGC 3256 , and IC 1623 is associated with moderately broadened emission-line profiles and enhanced emission-line ratios of lower ionization species such as [S II] $\lambda \lambda 6717,6731 \AA$ and [O I] $\lambda 6300 \AA$ (Monreal-Ibero et al. 2010; Rich et al. 2011). The shocks themselves are due to major gas flows caused by the merger process. During a major merger, gas is driven inward by tidal forces, which can result in shock excitation (e.g., Farage et al. 2010). This infalling gas fuels massive bursts of star formation and AGN activity, which in turn drive massive galactic outflows and further shocks into the ISM and beyond. In an effort to determine the contribution from shocks in U/LIRGs and the relationship between shocks and merger progress, we have searched for similar signatures in a larger IFS sample of 10 nearby U/LIRGs (Rich et al. 2012). We find that many of the galaxies in our sample, especially those in 
later-stage mergers, exhibit evidence of significant ongoing shock excitation in their optical spectra. To characterize the relative contribution from shocks throughout the merger process, we extend and expand on the analyses from our previous work in Rich et al. (2010, 2011) with a sample of U/ LIRGs covering the entire merger sequence.

This paper presents a comprehensive study of the emissionline properties of the Wide Field Spectrograph (WiFeS) Great Observatory All-Sky LIRG Survey (GOALS) sample (WiGS), with an emphasis on understanding the shock emission observed. The sample, observations, and analysis are described in Sections 2 and 3. The emission-line ratio maps and diagnostic diagrams for the sample are discussed in Section 4. A summary of the velocity dispersion distributions for the WiFeS GOALS sample is given in Section 5. Section 6 summarizes the various diagnostics as a function of merger stage. The total contribution from shocks as a function of merger stage, the potential impact of shock excited gas on observational interpretation, and the potential overlap with AGN/composite galaxies is discussed in Section 7. Our conclusions are presented in Section 8.

We use the cosmological parameters assumed in the summary of the GOALS sample of Armus et al. (2009), based on the five-year WMAP results of Hinshaw et al. (2009): $H_{0}=70 \mathrm{~km} \mathrm{~s}^{-1} \mathrm{Mpc}^{-1}, \Omega_{\mathrm{V}}=0.72$, and $\Omega_{\mathrm{M}}=0.28$.

\section{SAMPLE, OBSERVATIONS, AND DATA REDUCTION}

Our targets are U/LIRGs drawn from the GOALS sample (Armus et al. 2009). GOALS is a multiwavelength survey of the brightest $60 \mu \mathrm{m}$ extragalactic sources in the local universe $\left(\log \left(L_{\mathrm{IR}} / L_{\odot}\right)>11.0\right)$ with redshifts $z<0.088$. GOALS is a complete subset of the IRAS Revised Bright Galaxy Sample (RBGS; Sanders et al. 2003). Objects in GOALS cover the full range of nuclear spectral types and interaction stages and may serve as useful analogs for comparison with high-redshift galaxies.

The GOALS U/LIRGs represent an entire array of merger stages from isolated starburst systems to massive post-mergers, as well as galaxies in compact groups. The GOALS systems include starburst galaxies, AGNs, composite systems, LINERs, and E+a galaxies. While the ULIRGs in GOALS are composed entirely of major mergers, the lower-luminosity LIRGs represent a variety of systems-some past or future ULIRGs, others simply undergoing intense bursts of star formation driving up the infrared luminosity temporarily (Ishida 2004; Armus et al. 2009). Our sample consists of targets observable from the south.

\subsection{Observations}

Our observations were conducted with the Wide Field Field Spectrograph at the Mount Stromlo and Siding Spring Observatory $2.3 \mathrm{~m}$ telescope. WiFeS is a dual-beam, imageslicing integral field unit (IFU) described in detail by Dopita et al. (2007, 2010). We have previously presented analysis of smaller portions of the WiFeS GOALS data set in Rich et al. $(2010,2011,2012)$. In short, a single pointing from our data set provides a data cube $25^{\prime \prime} \times 38^{\prime \prime}$, sampled with $1^{\prime \prime}$ spaxels in double binning mode. The typical seeing was $\sim 1$ ". 5 . The setup used for our observations produces a spectral coverage of 3700-5700 $\AA$ for the blue spectra, at a spectral resolution of $R \sim 3000\left(100 \mathrm{~km} \mathrm{~s}^{-1}\right)$ and $5700-7000 \AA$ for the red spectra at
Table 1

WiFeS IFU GOALS Sample (WIGS)

\begin{tabular}{lcccc}
\hline \hline $\begin{array}{l}\text { IRAS Name } \\
\text { (a) }\end{array}$ & $\begin{array}{c}L_{\text {IR }} / L_{\odot} \\
\text { (b) }\end{array}$ & $\begin{array}{c}z \\
(\mathrm{c})\end{array}$ & $\begin{array}{c}\mathrm{pc} /{ }^{\prime \prime} \\
(\mathrm{d})\end{array}$ & $\begin{array}{c}\text { Merger Stage } \\
(\mathrm{e})\end{array}$ \\
\hline F01053-1746 & 11.71 & 0.0201 & 399 & $\mathrm{~b}$ \\
F02072-1025 & 11.01 & 0.0129 & 256 & $\mathrm{a}$ \\
F06076-2139 & 11.65 & 0.0374 & 742 & $\mathrm{~b}$ \\
08355-4944 & 11.62 & 0.0259 & 543 & cde \\
F10038-3338 & 11.78 & 0.0341 & 707 & cde \\
F10257-4339 & 11.64 & 0.0094 & 185 & $\mathrm{~b}$ \\
F12043-3140 & 11.43 & 0.0232 & 517 & $\mathrm{~b}$ \\
F12592+0436 & 11.68 & 0.0375 & 784 & cde \\
13120-5453 & 12.32 & 0.0308 & 653 & cde \\
F13373+0105 & 11.62 & 0.0226 & 502 & $\mathrm{a}$ \\
F15107+0724 & 11.35 & 0.0130 & 308 & iso \\
F16164-0746 & 11.62 & 0.0272 & 588 & cde \\
F16399-0937 & 11.63 & 0.0270 & 584 & $\mathrm{~b}$ \\
F16443-2915 & 11.37 & 0.0209 & 465 & $\mathrm{a}$ \\
F17138-1017 & 11.49 & 0.0173 & 392 & cde \\
F17207-0014 & 12.46 & 0.0428 & 878 & cde \\
F17222-5953 & 11.41 & 0.0208 & 456 & iso \\
17578-0400 & 11.48 & 0.0140 & 322 & a \\
F18093-5744 & 11.62 & 0.0173 & 383 & b \\
F18293-3413 & 11.88 & 0.0182 & 401 & b \\
F18341-5732 & 11.35 & 0.0156 & 344 & iso \\
F19115-2124 & 11.93 & 0.0487 & 976 & b \\
F20551-4250 & 12.06 & 0.043 & 860 & cde \\
F21330-3846 & 11.14 & 0.0191 & 400 & b \\
F21453-3511 & 11.42 & 0.0162 & 341 & iso \\
F22467-4906 & 11.84 & 0.0430 & 853 & cde \\
F23128-5919 & 12.06 & 0.0446 & 884 & $\mathrm{~b}$ \\
\hline & & & & \\
\hline
\end{tabular}

Notes.

(a) IRAS identifier from Sanders et al. (2003) and Armus et al. (2009); (b) log $\left(L_{\mathrm{IR}} / L_{\odot}\right.$ ) (Sanders et al. 2003; Armus et al. 2009); (c) systemic redshift (Sanders et al. 2003); (d) spatial scale; (e) Veilleux \& Rupke (2002) merger stage, taken from Yuan et al. (2010) where possible.

a resolution of $R \sim 7000\left(40 \mathrm{~km} \mathrm{~s}^{-1}\right)$. Thus, the data have sufficient spectral resolution in the red to allow detailed dynamical studies, while the total wavelength coverage (3700-7000 $\AA$ ) is sufficient to support excitation and chemical abundance analyses.

The data were taken over five separate observing runs in 2009 July, August, and September and 2010 March and May. Observations were begun shortly after the instrument was commissioned, prior to the availability of nod-and-shuffle mode. Hence, data taken in 2009 are taken primarily in classical observing mode with separate observations of the sky throughout each evening, while observations carried out in 2010 primarily use nod-and-shuffle mode for sky subtraction. Tables 1 and 2 list the systems observed and their properties. Table 2 denotes the dates observed, with the number of individual WiFeS pointings and total exposure times for each pointing, divided among two to five exposures. Figure 12 shows our WiFeS pointings overlaid on Digitized Sky Survey (DSS) images for each system listed in Table 2. We observe a total of 27 GOALS systems with a wide variety of properties described in Section 2.3. Observations were generally aligned and/or mosaicked to cover the entire galaxy or galaxies in each system.

\subsection{Data Reduction}

The data were reduced and flux calibrated using the WiFeS pipeline, briefly described in Dopita et al. (2010), which uses 
Table 2

WiFeS Observations

\begin{tabular}{|c|c|c|c|c|c|}
\hline $\begin{array}{l}\text { IRAS Name } \\
\text { (a) }\end{array}$ & $\begin{array}{l}\text { Optical ID } \\
\text { (b) }\end{array}$ & $\begin{array}{l}\text { Observation Date } \\
\text { (c) }\end{array}$ & $\begin{array}{c}\text { Pointing Center (J2000) } \\
\text { (d) }\end{array}$ & $\begin{array}{l}\text { PA (deg) } \\
\quad(\mathrm{e})\end{array}$ & $\begin{array}{r}\text { Exp. (minutes) } \\
\text { (f) }\end{array}$ \\
\hline F19115-2124 & ESO 593-IG008 & 2009 Jul 26 & $19^{\mathrm{h}} 14^{\mathrm{m}} 30^{\mathrm{s}} .8-21^{\mathrm{d}} 19^{\mathrm{m}} 05^{\mathrm{s}}$ & 305 & 120 \\
\hline F16443-2915 & ESO 453-G005 & 2009 Jul 28 & $16^{\mathrm{h}} 47^{\mathrm{m}} 31^{\mathrm{s}} \cdot 1-29^{\mathrm{d}} 21^{\mathrm{m}} 22^{\mathrm{s}}$ & \multicolumn{2}{|l|}{$\begin{array}{l}115 \\
115\end{array}$} \\
\hline \multirow[t]{2}{*}{ F23128-5919 } & ESO 148-IG002 & 2009 Jul 28 & $23^{\mathrm{h}} 15^{\mathrm{m}} 47^{\mathrm{s}} .1-59^{\mathrm{d}} 03^{\mathrm{m}} 25^{\mathrm{s}}$ & 173 & 67 \\
\hline & & 2009 Aug 14, 15 & $23^{\mathrm{h}} 15^{\mathrm{m}} 46^{\mathrm{s}} .7-59^{\mathrm{d}} 03^{\mathrm{m}} 00^{\mathrm{s}}$ & 173 & 59 \\
\hline F18293-3413 & $\cdots$ & 2009 Aug 14 & $18^{\mathrm{h}} 32^{\mathrm{m}} 41^{\mathrm{s}} \cdot 1-34^{\mathrm{d}} 11^{\mathrm{m}} 26^{\mathrm{s}}$ & 130 & 84 \\
\hline F18341-5732 & IC 4734 & 2009 Aug 18 & $18^{\mathrm{h}} 38^{\mathrm{m}} 24^{\mathrm{s}} .5-57^{\mathrm{d}} 29^{\mathrm{m}} 27^{\mathrm{s}}$ & 10 & 60 \\
\hline & & 2009 Aug 18 & $18^{\mathrm{h}} 38^{\mathrm{m}} 27^{\mathrm{s}} \cdot 0-57^{\mathrm{d}} 29^{\mathrm{m}} 28^{\mathrm{s}}$ & 10 & 60 \\
\hline F22467-4906 & ESO 239-IG002 & 2009 Aug 18 & $22^{\mathrm{h}} 49^{\mathrm{m}} 40^{\mathrm{s}} \cdot 6-48^{\mathrm{d}} 51^{\mathrm{m}} 00^{\mathrm{s}}$ & 30 & 40 \\
\hline & & 2009 Aug 18 & $22^{\mathrm{h}} 39^{\mathrm{m}} 39^{\mathrm{s}} \cdot 0-48^{\mathrm{d}} 50^{\mathrm{m}} 51^{\mathrm{s}}$ & 30 & 40 \\
\hline F02072-1025 & NGC 839 & 2009 Aug 18 & $02^{\mathrm{h}} 09^{\mathrm{m}} 42^{\mathrm{s}} \cdot 6-10^{\mathrm{d}} 10^{\mathrm{m}} 49^{\mathrm{s}}$ & 350 & 40 \\
\hline & & 2009 Aug 18 & $02^{\mathrm{h}} 09^{\mathrm{m}} 42^{\mathrm{s}} .8-10^{\mathrm{d}} 11^{\mathrm{m}} 13^{\mathrm{s}}$ & 350 & 40 \\
\hline F06076-2139 & $\ldots$ & 2010 Mar 12,13 & $06^{\mathrm{h}} 09^{\mathrm{m}} 45^{\mathrm{s}} \cdot 9-21^{\mathrm{d}} 40^{\mathrm{m}} 30^{\mathrm{s}}$ & 170 & 60 \\
\hline 08355-4944 & $\ldots$ & 2010 Mar 14,15 & $08^{\mathrm{h}} 37^{\mathrm{m}} 02^{\mathrm{s}} \cdot 2-49^{\mathrm{d}} 54^{\mathrm{m}} 36^{\mathrm{s}}$ & 135 & 80 \\
\hline $13120-5453$ & $\ldots$ & 2010 Mar 14,16 & $13^{\mathrm{h}} 15^{\mathrm{m}} 06^{\mathrm{s}} 3-55^{\mathrm{d}} 09^{\mathrm{m}} 26^{\mathrm{s}}$ & 15 & 95 \\
\hline F10257-4339 & NGC 3256 & 2010 Mar 15 & $10^{\mathrm{h}} 27^{\mathrm{m}} 51^{\mathrm{s}} \cdot 9-43^{\mathrm{d}} 54^{\mathrm{m}} 10^{\mathrm{s}}$ & 165 & 60 \\
\hline & & 2010 Mar 15 & $10^{\mathrm{h}} 27^{\mathrm{m}} 50^{\mathrm{s}} .1-43^{\mathrm{d}} 54^{\mathrm{m}} 15^{\mathrm{s}}$ & 165 & 60 \\
\hline F13373+0105 & Arp 240 & 2010 Mar 15,16 & $13^{\mathrm{h}} 39^{\mathrm{m}} 52^{\mathrm{s}} .8+00^{\mathrm{d}} 50^{\mathrm{m}} 26^{\mathrm{s}}$ & 35 & 60 \\
\hline & & 2010 May 7 & $13^{\mathrm{h}} 39^{\mathrm{m}} 57^{\mathrm{s}} .8+00^{\mathrm{d}} 49^{\mathrm{m}} 56^{\mathrm{s}}$ & 130 & 50 \\
\hline F10038-3338 & ESO 374-IG032 & 2010 Mar 16 & $10^{\mathrm{h}} 06^{\mathrm{m}} 04^{\mathrm{s}} .5-33^{\mathrm{d}} 53^{\mathrm{m}} 10^{\mathrm{s}}$ & 45 & 60 \\
\hline $\mathrm{F} 12592+0436$ & CGCG 043-099 & 2010 Mar 16 & $13^{\mathrm{h}} 01^{\mathrm{m}} 50.3+04^{\mathrm{d}} 20^{\mathrm{m}} 04^{\mathrm{s}}$ & 75 & 40 \\
\hline F12043-3140 & ESO 440-IG058 & 2010 May 7,8 & $12^{\mathrm{h}} 06^{\mathrm{m}} 51^{\mathrm{s}} \cdot 7-31^{\mathrm{d}} 56^{\mathrm{m}} 55^{\mathrm{s}}$ & 60 & 100 \\
\hline F16164-0746 & $\ldots$ & 2010 May 7 & $16^{\mathrm{h}} 19^{\mathrm{m}} 11^{\mathrm{s}} \cdot 6-07^{\mathrm{d}} 54^{\mathrm{m}} 03^{\mathrm{s}}$ & 60 & 75 \\
\hline F18093-5744 & IC 4687 & 2010 May 7, 13 & $18^{\mathrm{h}} 13^{\mathrm{m}} 40^{\mathrm{s}} .1-57^{\mathrm{d}} 43^{\mathrm{m}} 28^{\mathrm{s}}$ & 50 & 116 \\
\hline
\end{tabular}

Note.

(a) IRAS identifier from Sanders et al. (2003) and Armus et al. (2009); (b) optical counterpart from Armus et al. (2009); (c) observation dates; (d) center of pointing; (e) position angle, degrees $\mathrm{E}$ of $\mathrm{N}$; (f) total pointing exposure time in minutes.

IRAF routines adapted primarily from the Gemini NIFS data reduction package. Cosmic-ray removal was performed on the raw data frames prior to reduction with the "dcr" routine (Pych 2004).

The bias subtraction is somewhat complicated by the use of quad-readout with four amplifiers on each CCD to decrease chip readout time. Additionally, there is a slight slope and instability in the bias across each region of the chip. Bias frames are taken immediately before and after each set of observations, and a 2D fit of the surface is subtracted from the temporally nearest object data in order to avoid adding additional noise to the data. Any resulting residual is accounted for with a fit to unexposed regions of the detector.

Quartz lamp flats are used to account for the response curve of the chip, and twilight sky flats are used to correct for illumination variation along each of the slitlets. Spatial calibration is carried out by placing a thin wire in the filter wheel and illuminating the slitlet array with a continuum lamp.
This procedure defines the center of each slitlet. The individual spectra have no spatial distortion because the camera corrects the small amount of distortion introduced by the spectrograph. Thus, only low-order spatial mapping of the slitlets is required.

Wavelength calibration is performed using $\mathrm{CuAr}$ and $\mathrm{NeAr}$ arc lamp observations to provide sufficient lines in both the blue and red arms of the camera. Arc lamp data were taken in between sets of object exposures. Each of the 25 slitlets is then rectified by the pipeline into a full data cube (one for each camera) sampled on a common wavelength scale.

Telluric absorption features were removed from the resulting red data cubes using observations of B stars or featureless white dwarfs (typically also used as flux standards) taken at similar air mass. The effects of differential atmospheric refraction are calculated and corrected by the pipeline for the blue data cubes.

Flux calibration of each individual data cube was carried out using flux standards observed throughout each night. Standard 
stars are taken from the WiFeS observing manual (Bessell 1999). Individual data cubes were flux calibrated using the standards observed nearest in time and air mass.

The individual data cubes are binned by 2 pixels in the spatial direction to increase signal-to-noise ratio $(\mathrm{S} / \mathrm{N})$ and produce square spatial pixels (spaxels) $1^{\prime \prime} \times 1^{\prime \prime}$. Observations taken in 2009 March were binned during the data reduction process; observations taken in 2009 May and later were binned on-chip. Typical seeing achieved at Siding Spring Observatory (SSO) during our observations is $\sim 1 " .5$, with some variation, on par with the spaxel size for our data cubes. Individual data cubes thus consist of $25 \times 381^{\prime \prime}$ square spaxels, producing over 900 spectra. In practice, a few rows of spaxels are trimmed from the top and bottom of the individual cubes, corresponding to the edges of the slitlets.

Finally, the individual reduced, flux-calibrated data cubes are median combined and sampled to a common spatial grid using overlapping features found in each pointing. This is done for both single pointing and mosaicked observations. Combined data cubes were aligned astrometrically by comparing a pseudo " $r$-band" image generated using the red spectrum from each spaxel with either DSS R-band or Hubble Space Telescope (HST) data when available.

\subsection{The WiFeS GOALS Sample}

In all, 27 systems comprising nearly 40 individual galaxies were observed. The sample was selected to cover a range of infrared luminosities, merger stages, and nuclear activity. Basic sample properties are shown in Table 1.

The final sample comprises 4 ULIRGs and 23 LIRGs. This represents a slightly larger fraction of ULIRGs than the GOALS sample itself, which has 23 ULIRGs and 198 LIRGs. We apply the merger stage classification used in Yuan et al. (2010), adopted from Veilleux \& Rupke (2002). Isolated systems ("iso") have no neighbors within a projected distance of $100 \mathrm{kpc}$, wide pairs ("a") are separated by a distance of greater than $10 \mathrm{kpc}$, close pairs ("b") are separated by less than $10 \mathrm{kpc}$, and latest-stage, coalesced mergers are a combination of the original "diffuse," "compact," and "old" merger class ("cde")_-defined by an increase in compactness in $K$ band and decreasing to no evidence of any tidal features. This somewhat coarse sampling of the merger process is necessary given the small number in each class: two isolated systems, five wide pairs, 10 close pairs, and nine late-stage mergers. Nonetheless, we are still able to trace bulk changes in the properties of our sample as a function of merger stage. Individual notes about each system are provided in Appendix A.

To avoid contamination by AGN ionization in our analysis of shocks, four galaxies with strong optical, mid-IR, and X-ray AGN signatures are removed from the various merger stage bins and treated separately. The isolated galaxy IRAS F214533511 , the closely interacting pair IRAS F23128-5919, and the coalesced mergers IRAS 13120-5453 and F16164-0746 are all dominated by an AGN, as detected via [Ne v] in mid-IR Spitzer spectroscopy (Petric et al. 2011) and X-ray color/spectroscopy from Chandra observations (Farrah et al. 2007; Iwasawa et al. 2009, 2011). These galaxies are not included in their respective merger classes for the analysis of shocks for the remainder of the paper. The possibility of lower-level AGN activity in other systems is further discussed in Section 7.3.

Once the AGNs are removed, there are three isolated galaxies ("iso"), four close pairs ("a"), nine close pairs ("b"), and seven coalesced systems ("cde"). The average $\log \left(L_{\mathrm{IR}}\right)$ for the four classes are $11.37 \pm 0.04,11.37 \pm 0.26,11.62 \pm 0.23$, and $11.85 \pm 0.33 L_{\odot}$, respectively, and $11.86 \pm 0.4 L_{\odot}$ for the four AGNs.

\section{DATA ANALYSIS}

The key data products required for our work are accurate emission-line fluxes and emission-line kinematics at the highest possible spatial resolution, with as few confounding spectroscopic fitting parameters as possible. In order to tailor the final data product derived from the cubes to the needs of the analysis, in-house IDL routines combined with existing IDL software were used to analyze the spectra from each final data cube.

\subsection{Spectral Fitting}

Each data cube was analyzed using the UHSPECFIT software package (Rich et al. 2010; Rupke et al. 2010; Zahid et al. 2011). The WiFeS UHSPECFIT package cycles through the red and blue cubes for a single object, fitting the blue and red spectra from a given spaxel simultaneously. For each spectrum, the package performs an initial fit to the continuum and emission lines, checks whether any emission is found in the strongest lines ( $\mathrm{H} \alpha$ and $\left[\mathrm{N}_{\mathrm{II}}\right]$ ), and, if so, performs a second, final fit using the first fit as input estimators. After fitting every spectrum in the data cube in this fashion, the final data product is a data structure with spectral fits and all of the parameters necessary to reconstruct the emission-line component fits for each line of each spectrum, as well as the errors on those parameters. The details of the fitting routine are further described in the following subsections.

\subsection{Continuum Fitting}

The first step in fitting a spectrum in all cases was to fit and subtract a stellar continuum using stellar population synthesis models from González Delgado et al. (2005). This is done in order to obtain a pure emission-line spectrum while accounting more accurately for stellar features that underlie the emission lines in several systems. An IDL routine, IBACKFIT (Moustakas \& Kennicutt 2006), fits a linear combination of stellar templates to the stellar continuua. IBACKFIT employs a least-squares analysis using the Levenberg-Marquardt algorithm via MPFIT (Markwardt 2009). IBACKFIT was applied in each case only to the blue spectrum, which holds the majority of the stellar spectral features in the WiFeS data. The best-fitting combination of templates was then combined with a functional fit and extrapolated to the entire data cube. No information from stellar template fitting (i.e., stellar population age, metallicity, etc.) was derived for the work in this paper.

\subsection{Emission-line Fitting}

The continuum-subtracted spectrum is then fit with several Gaussian emission lines using routines built using the MPFIT package. The list of emission lines is chosen by the user prior to running UHSPECFIT. The emission lines fit in the WiFeS GOALS data include [O II] $\lambda \lambda 3727,3729, \mathrm{H} \epsilon, \mathrm{H} \delta, \mathrm{H} \gamma, \mathrm{H} \beta$, [O III] $\lambda \lambda 4959,5007$, [O I] $\lambda \lambda 6300,6364$, [N II] $\lambda \lambda 6548,6583$, $\mathrm{H} \alpha$, and [S II] $\lambda \lambda 6717,6731$. The [O II] and [S II] doublet flux ratios were allowed to vary within the upper and lower theoretical limits, and the flux ratios of the [O III], [O I $]$, and 


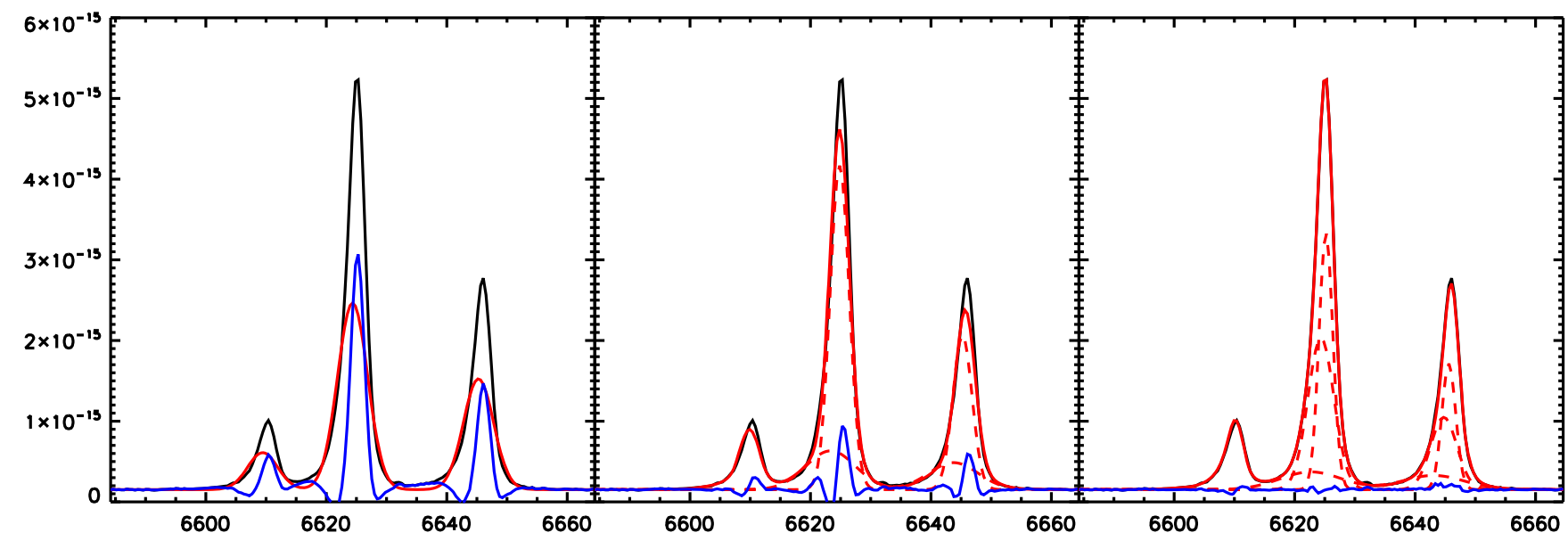

Figure 1. Example of one-, two-, and three-component fits applied to the $[\mathrm{N} \mathrm{II}]+\mathrm{H} \alpha$ complex prior to fitting the entire spectrum. This is an example of a spaxel from IRAS F10257-4339, where a three-component fit was chosen. The black line represents the data, the red line the best fit, and the blue line the residuals. Individual components are plotted with a dashed red line.

[N $\mathrm{N}$ II doublets were fixed to their prescribed theoretical values (i.e., Osterbrock 1989). Emission-line Gaussian peaks are constrained to be greater than or equal to zero.

One, two, or three Gaussian components are fit to every emission line, to account for the complex line profiles seen in several of the WiFeS GOALS systems. Prior to running UHSPECFIT on a galaxy, mpfit was used to fit one, two, and three Gaussian components to the $[\mathrm{N}$ II] $+\mathrm{H} \alpha$ lines. An example of this process is given in Figure 1. Each resulting one-, two-, and three-Gaussian-component fit was checked by eye. Upon inspection, the best fit was chosen based on both quantitative comparison of the respective $\chi^{2}$ and a qualitative assessment of the goodness of fit and consistency with features in surrounding fits within a single galaxy, similar to the method employed by Westmoquette et al. (2011, 2012).

If the $\chi^{2}$ does not markedly improve when an extra Gaussian component is added, fewer components are used to avoid overfitting the emission lines. Consideration is also given to emission-line fits in neighboring spaxels within a single cube when choosing whether to add additional Gaussian components. The best-fit emission-line widths and redshifts are then passed to UHSPECFIT, which holds them fixed while simultaneously varying the remaining parameters. The resulting emission-line fits to the rest of the spectrum were then again inspected by eye to ensure that poorly and erroneously fit lines are rejected. This method resulted in more reliable, consistent fits with UHSPECFIT.

All emission lines are fit simultaneously, but the redshift and velocity width of each Gaussian component are fixed to be the same value for every emission line. The relative velocities are fixed separately for the red and blue data cubes to allow for variation in wavelength calibration and resolution between the red and the blue spectra. This assumption implies that the lineemitting gas in a single spaxel is produced in the same region (e.g., Kewley et al. 2001b). Figure 2 shows an example of a successful multicomponent emission-line fit to key diagnostic lines in a single spectrum.

The errors in the parameters used in fitting the emission lines are calculated by the fitting code, which includes propagation of the variance spectra. The parameters include the Gaussian widths (velocity dispersions), ratio of dispersions between the blue and red spectra, individual Gaussian peaks, redshift, and any slight deviation between the blue and red wavelength calibrations.

\subsection{Data Products}

Once a galaxy has been run through UHSPECFIT, we generate maps of the recessional velocity, velocity dispersion, and emission-line fluxes of each emission-line component, as well as maps of the total emission-line flux of several strong lines. In addition to manual inspection of the emission-line fits, a final $\mathrm{S} / \mathrm{N}$ of 5 is used as a cutoff to create the total flux maps, and an $\mathrm{S} / \mathrm{N}$ of 3 cutoff as measured between component peaks and continuum noise is applied to the individual component maps. In all, $\sim 10,000$ spectra are analyzed in this paper, with $\sim 17,000$ individual Gaussian components above the $\mathrm{S} / \mathrm{N}$ cutoff. This corresponds to about 2100 individual Gaussian components in the "iso" galaxies, 3300 in "a," 8300 in "b," 1300 in "cde," and 1750 in the AGNs. The lower number of Gaussian components in the cde bin despite the higher number of galaxies reflects both the small spatial extent of the emission-line gas and the higher average distance to the coalesced merger galaxies.

\section{EMISSION-LINE GAS PROPERTIES}

Our emission-line maps and diagrams provide an overview of the distribution of spaxels dominated by enhanced low ionization species emission, as well as some indication of the underlying power source and metallicity. For our purposes, the maps and diagnostic diagrams are most useful in spatially separating regions dominated by $\mathrm{H}$ II region emission, shock emission, and AGN emission. The observed emission-line ratios are governed by the overall flux of ionizing photons, the hardness of the radiation field, and the metallicity of the gas. By taking emission-line ratios that are sensitive to abundance and with a variety of ionization potentials, the source of ionizing radiation can be determined (e.g., Baldwin et al. 1981; Veilleux \& Osterbrock 1987; Kewley et al. 2001a; Kauffmann et al. 2003; Kewley et al. 2006). With a harder radiation field than young stars or radiative shocks, AGN-ionized gas has generally higher $\log ([\mathrm{O} \mathrm{III}] / \mathrm{H} \beta)$ emission line ratios than $\mathrm{H}$ II region and shock emission, with $\log ([\mathrm{O} \mathrm{III}] / \mathrm{H} \beta)$ of order unity to a factor of several times unity. $\mathrm{H}_{\text {II }}$ regions exhibit a range of emission-line ratios, with low ionization species-to- 

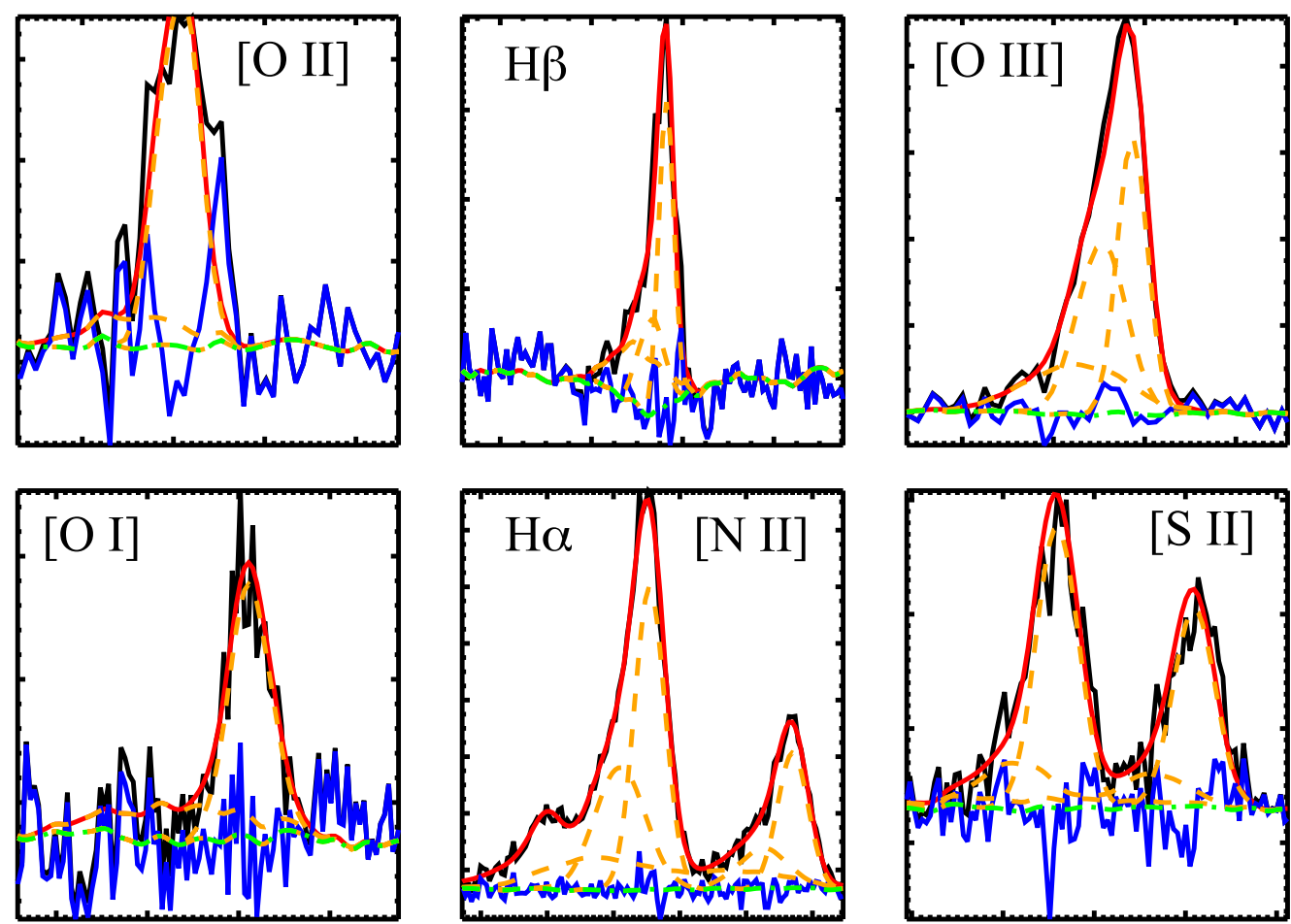

Figure 2. Example of a three-component fit to a spectrum from a single spaxel in IRAS F23128-5919. Several strong emission lines are shown. The total fit is plotted in red over the data in black, individual emission components are orange dashed lines, the continuum fit is a green dashed line, and the residual fit between the total fit and the emission fit is shown in blue. The individual component (orange lines) for the $[\mathrm{N}$ II] $\lambda 6548$ line has been removed to decrease confusion.

$\mathrm{H} \alpha$ ratios $\left(\log \left([\mathrm{N}\right.\right.$ II] $/ \mathrm{H} \alpha)<-0.4, \log \left(\left[\mathrm{S}_{\mathrm{II}}\right] / \mathrm{H} \alpha\right)<-0.3$, and $\left.\log \left(\left[\mathrm{O}_{\mathrm{I}}\right] / \mathrm{H} \alpha\right)<-1.0\right)$. $\mathrm{H}$ II regions show a large range of $\log$ $([\mathrm{O} \mathrm{III}] / \mathrm{H} \beta)$ emission line ratios $(\log ([\mathrm{O} \mathrm{III}] / \mathrm{H} \beta$ from -1.0 to 0.8 from high to low abundance, respectively) as a result of less efficient cooling leading to higher-ionization parameters and thus higher $[\mathrm{O} \mathrm{III}] / \mathrm{H} \beta$. Shocks with velocities of $\sim 100$ $200 \mathrm{~km} \mathrm{~s}^{-1}$, on the other hand, correspond to enhancements in lower-ionization lines ([N II], [S II], [O I]) and some enhancement in $[\mathrm{O} \mathrm{III}] / \mathrm{H} \beta$ in higher-metallicity systems (Rich et al. 2011).

\subsection{Distinguishing Power Sources}

Both the distribution of line ratios and the values of the line ratios themselves provide useful information about the source (s) of ionizing radiation in a galaxy. Emission-line ratio maps are generated using the total line fluxes spaxel by spaxel, which effectively traces the dominant power source. Line ratio diagnostic diagrams provide a more detailed perspective of the sources of ionizing radiation at work in a given system (Baldwin et al. 1981; Veilleux \& Osterbrock 1987; Kewley et al. 2001a, 2006; Kauffmann et al. 2003). When combined with the spatial information from the line ratio maps, a clear picture of one or more power sources present in a galaxy is revealed.

Figure 3 shows how the diagrams and line ratio maps appear for three systems with very different sources of ionizing radiation. Some of the galaxies in our sample appear to be dominated entirely by $\mathrm{H}$ II region emission, with no indication of any other source of ionizing radiation. IRAS F17222-5953 is a good example of such a system, as seen in Figure 3. The line ratios fall entirely within the $\mathrm{H}$ II region portion of the diagnostic diagram. The $[\mathrm{O}$ III $] / \mathrm{H} \beta$ and $[\mathrm{N}$ II] $/ \mathrm{H} \alpha$ ratios fall as a function of radius owing to the presence of a metallicity gradient (Rich et al. 2012). The gradient and line ratios are entirely consistent with other star-forming galaxies (Kewley et al. 2010; Rupke et al. 2010).

Some of the galaxies in our parent sample exhibit a blend of strong AGN activity with star formation. IRAS F21453-3511, shown in Figure 3, is a good example of this behavior. The diagnostic diagrams in these galaxies have very strong [O III]/ $\mathrm{H} \beta$, moving into the Seyfert/AGN portion of the diagnostic diagram. Line ratio maps tend to show the highest emission-line ratios in the nucleus, corresponding to the AGNs, decreasing with distance from the nucleus (Davies et al. 2014; Leslie et al. 2014).

Finally, some galaxies show evidence of star formation blended with composite/LINER-like emission. This is shown in Figure 3 with the close pair IRAS F01053-3511, which shows extended LINER-like emission well outside the nuclear regions, which are dominated primarily by star formation. Extended composite/LINER emission alone, however, does not confirm the presence of shocks (Rich et al. 2010, 2011). Extended lowionization emission regions (LIERs) can be caused by a number of mechanisms, including heating by post-AGB stars (Sarzi et al. 2010; Annibali et al. 2010; Eracleous et al. 2010) and diffuse ionized emission (e.g., Blanc et al. 2009).

\subsection{Line Ratio Maps}

Example emission-line ratio maps are shown in Figure 3, with the remaining galaxy emission-line maps given in the appendix. Emission-line ratio maps are generated using the total line fluxes spaxel by spaxel, which effectively traces the dominant power source. For instance, in Figure 3 large portions of the merging system IRAS F01053-1746 are dominated by emission-line ratios consistent with star formation, while there is evidence of enhanced emission-line ratios in areas of lower 

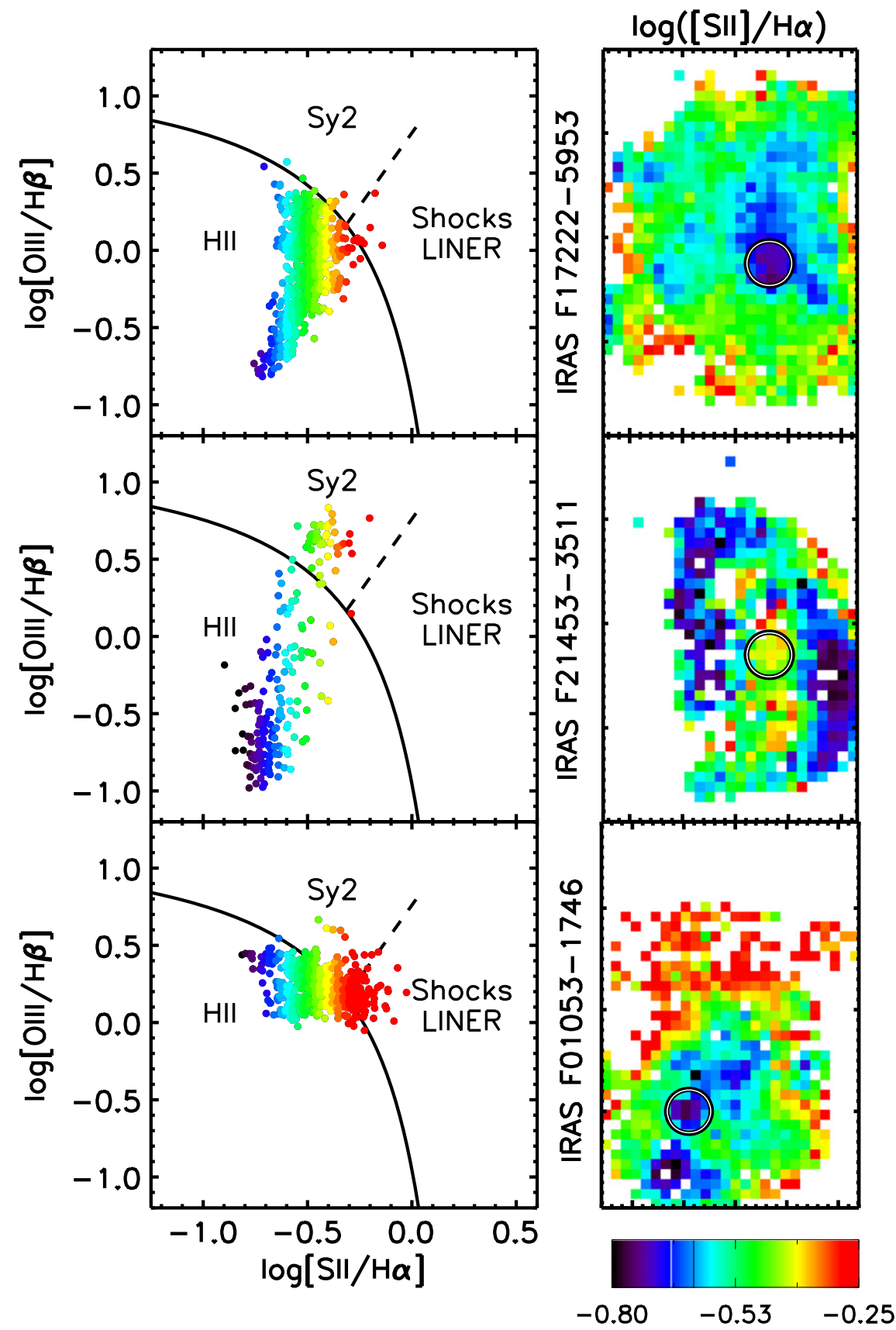

Figure 3. Example line ratio diagnostic diagrams and maps from IRAS F17222-5953, F21453-3511, and F01053-1746. Points in the diagnostic diagrams are color coded with respect to the $[\mathrm{S}$ II] $/ \mathrm{H} \alpha$ values shown in the line ratio maps. The center of each galaxy, corresponding to the peak in $R$-band emission, is marked with a circle in the line ratio map for reference. IRAS F17222 is dominated by star formation, F21453 is an AGN blended with star formation (Davies et al. 2014), and F01053 is a mixture of radiative shocks and star formation (Rich et al. 2011). These three galaxies are chosen to show key features of galaxies in our sample with each source of ionizing radiation. The remaining diagnostic diagrams and maps are shown in Appendix A.

$\mathrm{H} \alpha$ surface brightness (as traced by the contours). As is noted in Rich et al. (2011), even in some portions of IRAS F010531746 dominated by $\mathrm{H}_{\text {II }}$ region emission there may be a contribution to the emission from shocks that can be separated kinematically, but the total flux will show H II-region-like line ratios overall.

Systems dominated entirely by $\mathrm{H}$ II region emission include IRAS F13373+0105 W and E, IRAS F17222-5953, and IRAS F16443-2915 $\mathrm{N}$ and $\mathrm{S}$. These systems exhibit line ratios consistent with $\mathrm{H}$ II region emission (see also Section 4.2) across the entire line ratio map, with no indication of any other significant source of ionizing flux.
In some systems the line ratio map provides an immediate indication that there may be an AGN present. In systems with a strong AGN, the nuclear regions tend to be dominated by strong line ratios in every map, including $[\mathrm{O} \mathrm{III}] / \mathrm{H} \beta$. We are able to resolve and separate regions of star formation and AGN ionization in the maps of IRAS F21453-3511 and IRAS F23128-5919, both of which show a combination of Seyfert II nuclei and ongoing star formation (Davies et al. 2014; Leslie et al. 2014).

In many of the emission-line maps, galaxies show enhanced low-ionization line ratios away from the nucleus and in intergalaxy regions with $\mathrm{H}$ II-region-like line ratios in the 
nuclear regions: this radial rise in line ratios is opposite to the drop in line ratios seen in AGN-dominated systems. As shown in Rich et al. (2010, 2011), underlying shock excitation combined with a lower contribution from star formation with increasing radius reveals itself as stronger low-ionization line ratios away from the nucleus. Some examples include IRAS F12043-3140, IRAS F15107+0724, IRAS F17138-1017, IRAS F20551-4250, and IRAS F21330-384. In some cases this may be enhanced by galactic winds (Sharp \& BlandHawthorn 2010), as in IRAS F02072-1025 and IRASF F10257-4339 (Rich et al. 2010, 2011). Dust lanes also serve to bury ongoing star formation, enhancing shock-like line ratios, especially where shocks are caused by outflowing gas, the passage of dense clouds of gas through a substrate of more diffuse ISM, or through cloud-cloud collisions. This is evident in IRAS F01053-1746, IRAS F10257-4339, and IRAS F18293-3413. Some of the latest-stage mergers are entirely dominated by enhanced line ratios consistent with shock excitation, such as IRAS F12592+0436 and IRAS F224674906. Shocks are not restricted to portions of the galaxy dominated by shock emission, however, and can be spread across merging galaxies even where the optical emission is dominated by star formation (Rich et al. 2011).

\subsection{Line Ratio Diagnostic Diagrams}

Line ratio diagnostic diagrams provide a more detailed perspective of the sources of ionizing radiation at work in a given system (Baldwin et al. 1981; Veilleux \& Osterbrock 1987; Kewley et al. 2001a; Kauffmann et al. 2003; Kewley et al. 2006). When combined with the spatial information from the line ratio maps, a clearer picture of the combined power sources is revealed. Figure 3 shows example line diagnostic diagrams using the total emission-line flux for three galaxies with disparate sources of ionizing radiation including starforming regions, AGNs, and radiative shocks. The diagrams for the remaining 30 data cubes, including diagrams with individual line components rather than total flux, are shown in the Appendix.

Traditionally, emission-line ratio diagnostic diagrams have been applied to nuclear spectra of U/LIRGs to investigate the presence of AGNs (e.g., Yuan et al. 2010). Systems with resolved AGN activity provide a good example of the power of IFS. IRAS F21453-3511 has a cluster of spaxels in the AGN portion of all three diagnostic diagrams corresponding to the central regions seen in the emission-line ratio maps. The rest of the spaxels form a sequence stretching toward the $\mathrm{H}$ II region portion of the diagnostic diagrams as the line ratios decrease away from the nucleus.

The combination of line ratio maps and diagnostic diagrams provides a way to disambiguate the difference between AGNenhanced emission and shock-enhanced line ratios. Rich et al. (2010, 2011) discuss in detail this method for the systems IRAS F02072-1025, F01053-1746, and F10257-4339, but the same effect can be seen in, for example, IRAS F12043-3140 and IRAS F21330-3846. The latter two systems show a sequence of spaxels in the line diagnostic diagrams, indicating enhanced line ratios consistent with some contribution from non-H II region emission. The enhanced line ratios in the emission-line maps of these systems are not consistent with AGNs but with shock excitation due to the merger process.

As described in Rich et al. (2011), the presence of slow shocks leads to LINER-like line ratios in the diagnostic diagrams. The $\left[\mathrm{S} \mathrm{II}_{\mathrm{II}} / \mathrm{H} \alpha\right.$ and $\left[\mathrm{O}_{\mathrm{I}}\right] / \mathrm{H} \alpha$ ratios in particular are enhanced in the presence of shocks owing to their low ionization potential, as is seen in great detail in IRAS F010531746 and F10257-4339 (Rich et al. 2011). LINER-like line ratios are seen in extranuclear regions in some other systems as well (e.g., IRAS F20551-4250). Two of our systems, IRAS F13373+0105 and F18341-5732, exhibit evidence of nuclear LINER activity, which is more likely associated with the traditional low-luminosity AGN explanation for LINER emission, whether it be photoexcited (Ferland \& Netzer 1983; Halpern \& Steiner 1983; Ho 1999, 2009; Kewley et al. 2006; Eracleous et al. 2010) or shock excited (Fosbury et al. 1978; Baldwin et al. 1981; Dopita et al. 2015).

The underlying metallicity of the gas also affects the line ratios seen in the diagnostic diagrams. IRAS F17222-5953 and $13373+0105 \mathrm{~W}$ are good examples of this effect: both galaxies have an intrinsic metallicity gradient that creates the sequence of values seen in the $[\mathrm{N} \mathrm{II}] / \mathrm{H} \alpha$ diagrams (Rich et al. 2012). Most of the galaxies that show $\mathrm{H}_{\text {II }}$ region emission have spaxels that correspond to intrinsically high metallicity gas, with the exceptions being IRAS F01053-1746 and F18093$5744 \mathrm{C}$ and $\mathrm{N}$.

\section{VELOCITY DISPERSIONS}

Velocity dispersion provides a separate, independent test for the presence of shock emission (Rich et al. 2011). Here we examine the velocity dispersions of the emission-line Gaussian components. Velocity dispersions associated with $\mathrm{H}$ in regions are typically a few tens of kilometers per second, while lowvelocity shocks and AGN line profiles can have velocity dispersions in excess of $100 \mathrm{~km} \mathrm{~s}^{-1}$ (Epinat et al. 2010; Rich et al. 2011). In their discussions of the high velocity dispersions in the SINS sample, Genzel et al. (2008) note that the turbulent star formation, shocks, and stellar feedback can drive up velocity dispersion as well (Efstathiou 2000; Silk 2001; Monaco 2004; Thompson et al. 2005; Dib et al. 2006). This is consistent with the correlation between average velocity dispersion and star formation surface density seen in, for instance, the sample of Green et al. (2010).

\subsection{Dispersion Distributions}

We analyze the distribution of velocity dispersions fit to the individual components in each system. An example histogram of velocity dispersions is shown in Figure 4, with the remaining systems shown in the appendix. The dispersion distribution is shown as both the fraction of the total number of profiles at a given $\sigma$ and a fraction of the total $F_{\mathrm{H} \alpha}$ in a given velocity bin. All Gaussian components detected in at least one emission line are included in the velocity dispersion distribution histograms.

In systems mainly dominated by star formation (e.g., IRAS F16443-2915 N/S, F17222-5953) the majority of the velocity dispersions are consistent with the expected $\sigma$ of a few tens of kilometers per second. As noted in Rich et al. (2010, 2011), galaxies with a significant contribution from shocks show a significant contribution in the range $\sigma=100-200 \mathrm{~km} \mathrm{~s}^{-1}$, consistent with the shock velocities derived from line ratios (e.g., IRAS F01053-1746, F02072-1025, F10257-4339; Rich et al. 2010, 2011). Many galaxies also exhibit a significant number of components with $\sigma$ between 40 and $90 \mathrm{~km} \mathrm{~s}^{-1}$, likely consistent with disk turbulence. The galaxies in our sample that show unambiguous AGN signatures also show 
IRAS F01053-1746
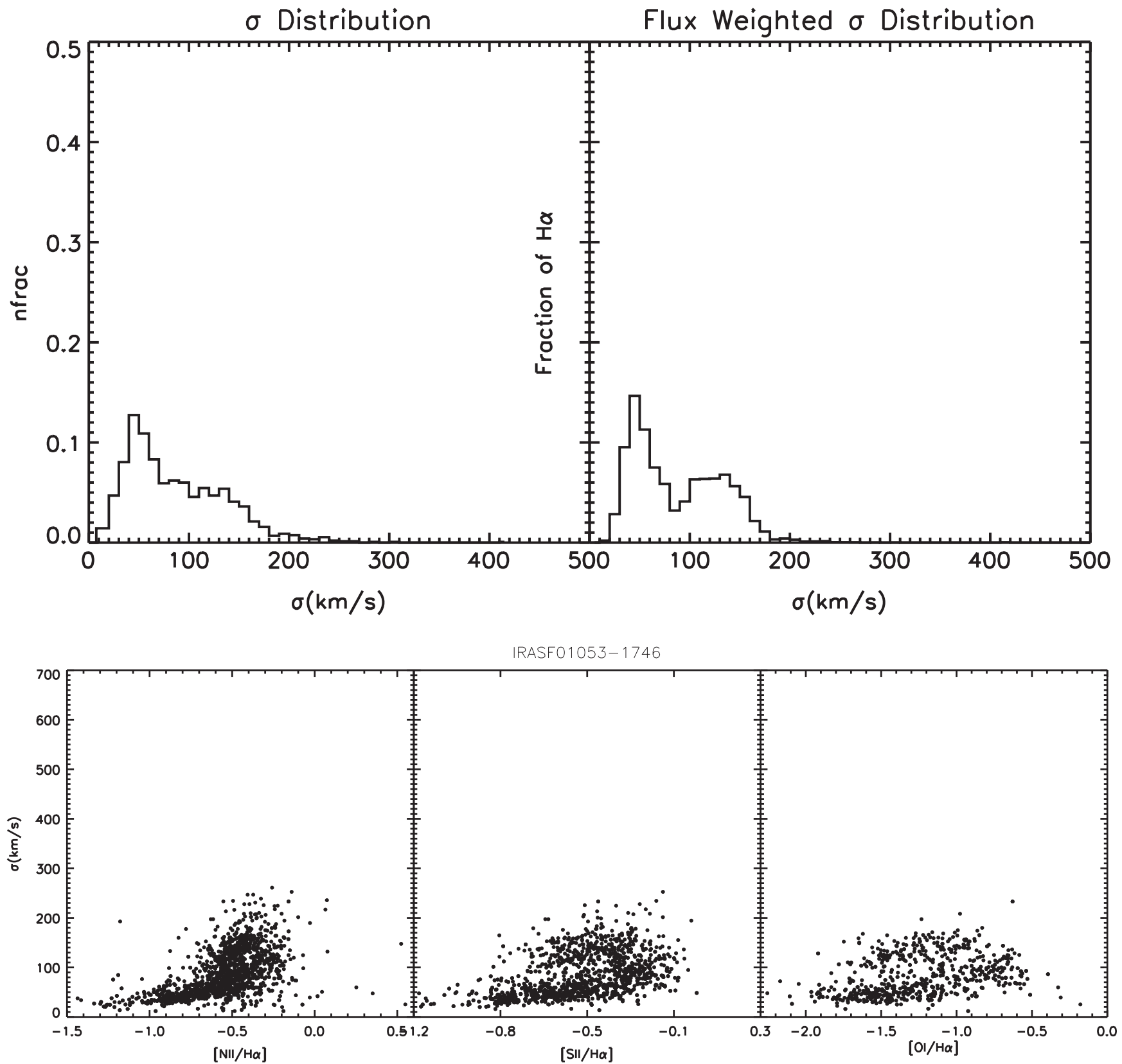

Figure 4. Velocity dispersion distribution and comparison with line ratios. The top left panel shows the histogram for the fraction of the total number of components for a given velocity dispersion. The top right panel shows a similar histogram, but with the fraction of the total $\mathrm{H} \alpha$ flux for a given velocity dispersion. Systems with a stronger contribution from shocks show a larger fraction of $\sigma \gtrsim 90 \mathrm{~km} \mathrm{~s}^{-1}$. The bottom three panels show various diagnostic emission-line ratios vs. velocity dispersion. Lower emission-line ratios are, on average, dominated by lower- $\sigma$ star-forming regions. The same diagrams for the remaining portion of the sample are in the appendix.

some contribution at significantly higher velocity dispersions, of $300-600 \mathrm{~km} \mathrm{~s}^{-1}$.

\subsection{Line Ratios and Velocity Dispersion}

In their lower spectral resolution IFS survey of U/LIRGs, Monreal-Ibero et al. (2006, 2010) established a correlation between velocity dispersion and emission-line ratio, which they attributed to shocked gas components. In Rich et al. (2011) we see the same correlation for IRAS F01053-1746 and F102574339 , consistent with the other signatures of shock excitation (Rich et al. 2011).
Nearly all of the galaxies in the WiFeS GOALS sample that have line profiles fit with elevated velocity dispersion components reproduce this enhancement in $[\mathrm{N}$ II $] / \mathrm{H} \alpha$, [S II]/ $\mathrm{H} \alpha$, and $\left[\mathrm{O}_{\mathrm{I}}\right] / \mathrm{H} \alpha$ with respect to velocity dispersion, regardless of the excitation mechanism. The exceptions to this rule appear to be IRAS F18093-5744 C and S. IRAS F18093$5744 \mathrm{C}$ in particular shows the opposite trend: a component with elevated velocity dispersion and correspondingly lower $\left[\mathrm{N}\right.$ II] $/ \mathrm{H} \alpha,\left[\mathrm{S}_{\mathrm{II}}\right] / \mathrm{H} \alpha$, and [O I] $/ \mathrm{H} \alpha$ ratios. IRAS F18093-5744 $\mathrm{C}$ is a compact Wolf-Rayet galaxy with the emission driven by young, bright stars (Kovo \& Contini 1999; Fernandes et al. 2004). Our data show two narrow components with an 
underlying broad component seen in the $\sigma$ versus ratio diagrams. This broader component is elevated in [O III]/ $\mathrm{H} \beta$ and can be seen in the diagnostic diagram in the Appendix, corresponding to lower-metallicity gas, consistent with the analysis in Fernandes et al. (2004).

\section{MERGER PROGRESS}

All line diagnostic methods described in Section 5 have been applied to each galaxy in our sample. We now track how they change, if at all, as a function of merger evolution. We group spaxels from galaxies in each of the four merger stages as outlined in Section 2. The four positively identified AGNs are placed in a separate group. We now examine the overall shape of the line ratio diagnostic diagrams, velocity dispersion distribution, and $\sigma$ versus line ratio diagrams for each merger stage.

\subsection{Diagnostic Diagram Evolution}

Figure 5 shows the three diagnostic diagrams for each merger group and the AGN. The points are generated using the total flux line ratios in each spaxel for every galaxy in a given merger class. In the case of the close pair stage, the data points are somewhat dominated by IRAS F01053-1746 and F102574339 , but the other individual galaxies in this merger stage group have line ratios that fall in consistent regions of the diagnostic diagram. The AGN stage is likewise dominated by IRAS F21453-3511 and F23128-5919, which have comparatively spaxels in their diagnostic diagrams, though the relatively fewer points from the other two AGNs are again consistent.

The overall shape of the diagnostic diagrams shows an increasing number of spaxels in the "composite" region of the $[\mathrm{N}$ II $] / \mathrm{H} \alpha$ line ratio diagnostic diagram in later merger stages. This result is consistent with the work of Yuan et al. (2010), which shows an increasing fraction of composite behavior with merger stage.

There is a much narrower range of spaxels in the $\mathrm{H}$ II region portion of the $[\mathrm{N} \mathrm{II}] / \mathrm{H} \alpha$ diagram for the "cde" galaxies, which is primarily a result of the flattening and dilution of metallicity gradients as mergers progress: we discuss this effect further in Rich et al. (2012). The same effect is seen in individual systems in the "b" stage.

\subsection{Velocity Dispersion Evolution}

As in the case of line ratio evolution with merger stage, there is also clear evidence of a corresponding evolution in the observed velocity dispersion. In Figure 6, we show the change in the overall velocity dispersion distribution as a function of merger stage. The isolated systems are dominated primarily by narrow $\mathrm{H}$ II-region-like velocity dispersions, though there is a tail of higher dispersion spaxels corresponding to some turbulence and the LINER emission in IRAS F18341-3413. In the widely separated pairs the peak in both number of profiles and total $\mathrm{H} \alpha$ flux shifts slightly to the right and the tail of broader spaxels grows, indicating an increase in turbulent star formation. Figure 7 compares the velocity dispersion distribution for the isolated, "cde," and AGN systems. The AGN galaxies show a stronger high- $\sigma$ component above $350 \mathrm{~km} \mathrm{~s}^{-1}$ that is not seen in the other distributions.

In the closely interacting pairs, the shape of the dispersion distribution begins to change significantly: a strong tail of higher- $\sigma$ line profiles is seen in both fractional number and total $F_{\mathrm{H} \alpha}$. This is consistent with the increase in outflows as the merger progresses, as seen in previous IFU studies (Rich et al. 2011; Soto et al. 2012; Arribas et al. 2014; Genzel et al. 2014; Ho et al. 2014; Wild et al. 2014) and in Section 5 above. At the latest merger stages, the velocity dispersion distribution is fairly flat from narrow to broad line components, indicating a significantly increased contribution from turbulent star formation and shocks in the emission-line gas.

Figure 8 shows the evolution of the line ratio versus $\sigma$ with merger stage. The overall shape of the diagrams tells the same story as the diagnostic diagrams and velocity dispersion distributions taken together: there is an overall trend in the total number of spaxels with enhanced velocity dispersion and emission-line ratio as a function of merger stage.

The AGNs show a bimodal distribution in the line ratio versus $\sigma$, owing mainly to the difference in line ratio values at high velocity dispersion between IRAS F21453-3511 and F23128-5919 that dominate these figures. This is likely due to a difference in metallicity between the AGN-illuminated gas in the two systems.

\section{DISCUSSION}

In the absence of an AGN, a relative increase in the velocity dispersion and shift of the strong emission line ratios in the diagnostic diagram toward the LINER region is indicative of gas excited by slow shocks (Monreal-Ibero et al. 2006, 2010; Rich et al. 2010, 2011). Moreover, high- $\sigma$ and LINER-like line components are not restricted to the nuclear regions of our nonAGN systems, again consistent with widespread shock excitation caused by merger-driven gas flows as the source of ionization for the observed emission (Monreal-Ibero et al. 2010; Rich et al. 2011; see also individual system notes in Appendix A). We therefore interpret the overall relative increase in velocity dispersion, particularly in the absence of any detectable influence from an AGN, as an increased relative contribution from slow shock excitation in our sample and discuss the relative strengths of each velocity component in our sample.

\subsection{Shock Fraction and Merger Stage}

With the simple correlations established in the previous sections, $\sigma$ can be used as a proxy for the total contribution from $\mathrm{H}$ il region emission, turbulent star formation, and shocks. The histograms of the distribution of $\sigma$ discussed in Section 5 and shown in Figures 6 and 7 allow us to establish approximate cutoffs between the three components. For the discussion here we establish an upper limit of $\sigma=50 \mathrm{~km} \mathrm{~s}^{-1}$ for pure $\mathrm{H}_{\text {II }}$ region emission and $\sigma>90 \mathrm{~km} \mathrm{~s}^{-1}$ for spaxels that are primarily dominated by shock excitation in the absence of an AGN.

Figure 9 shows the change in the total fraction of $F_{\mathrm{H} \alpha}$ in each component as a function of merger stage. The fraction in each component is the total of the profiles in each merger stage bin. There is a minimal flux-weighted contribution of a few percent from a higher- $\sigma$, shock-dominated component in the three isolated galaxies and in the widely separated pairs in our sample. The turbulent, mid-range $\sigma$ component increases as the galaxies begin to interact, but it stays roughly flat throughout the merger process. Most interestingly, however, is the rapid increase in a high- $\sigma$ component from wide to close pairs and 


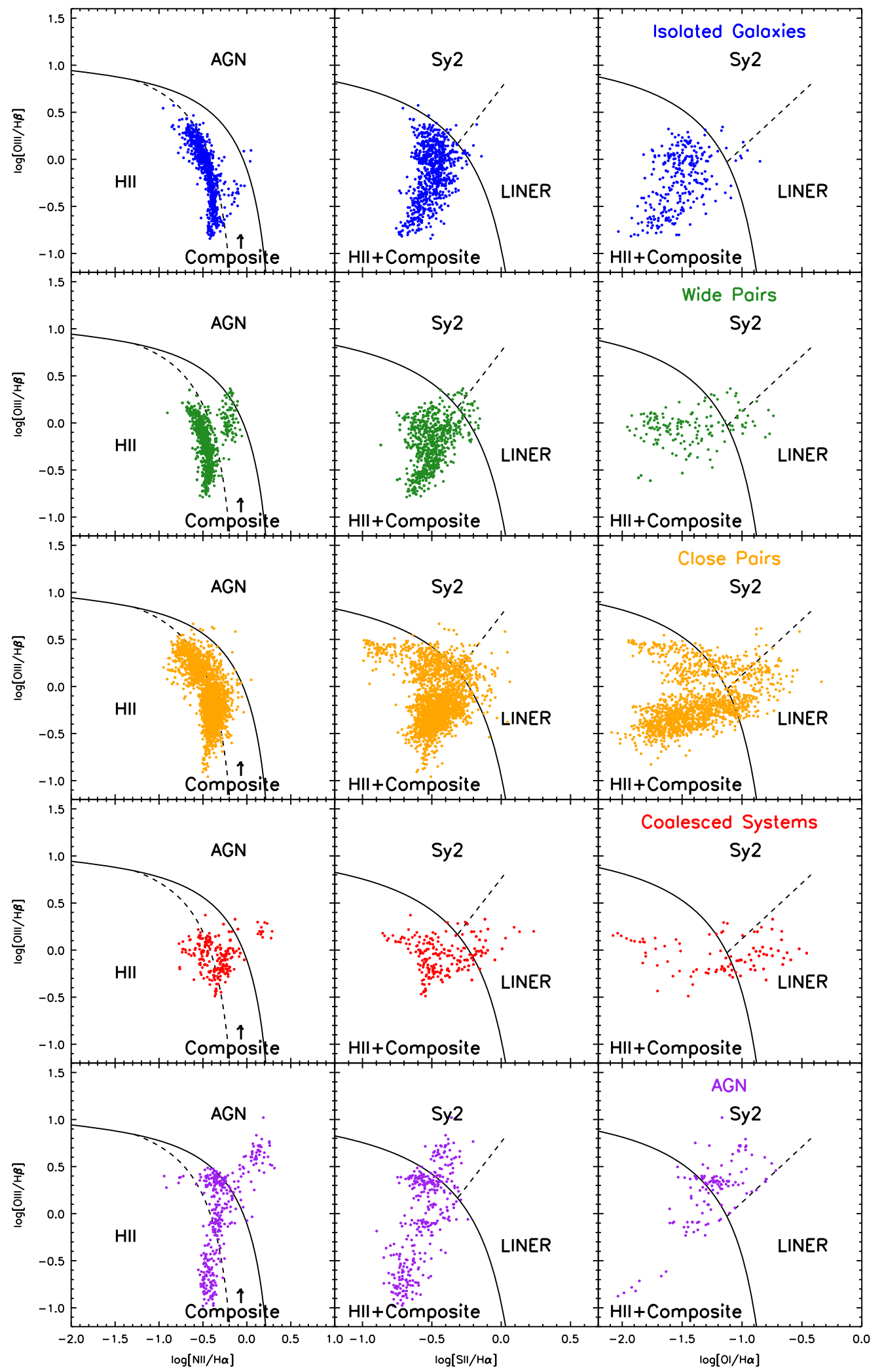

Figure 5. Line ratio diagnostic diagrams created using all spaxels from every galaxy in each merger class. In blue are isolated systems, green are widely separated pairs ("a"), orange are close pairs ("b"), red are coalesced galaxies ("cde"), and the bottom diagrams with purple points are the four AGN galaxies. Kewley et al. (2006) classifications are shown on each diagram.

from close pairs to late-stage mergers. By the "cde" stage, just over half of the total $F_{\mathrm{H} \alpha}$ we measure lies in high velocity dispersion line emission.
The low- $\sigma$ component is consistent with the velocity dispersions seen in star-forming regions, while the mid- $\sigma$ component is likely associated with regions of more turbulent 


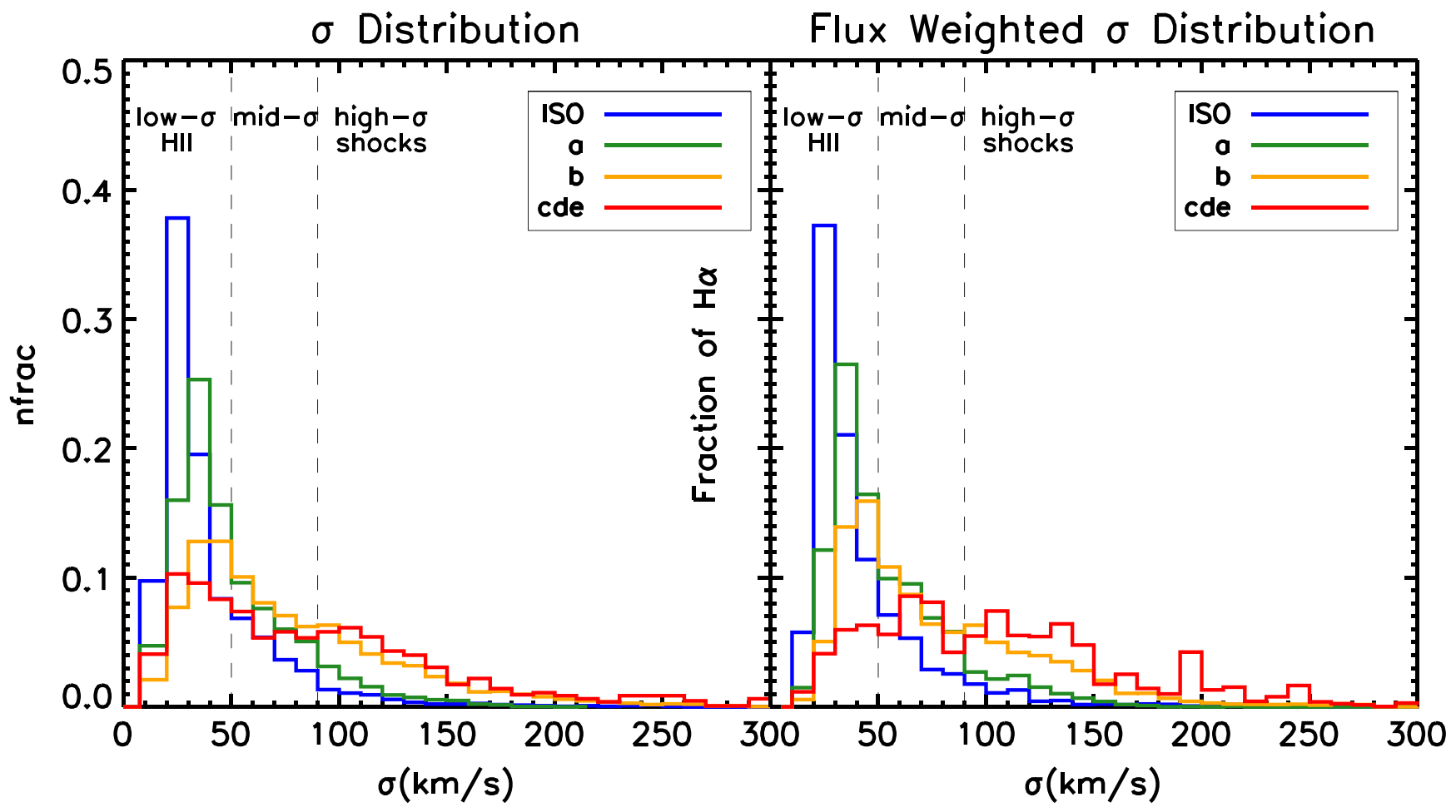

Figure 6. Velocity dispersion distribution histograms for each merger class. As in Figure 4, the fractional number of profiles is shown in the left panel and fluxweighted $\sigma$ distribution in the right panel.

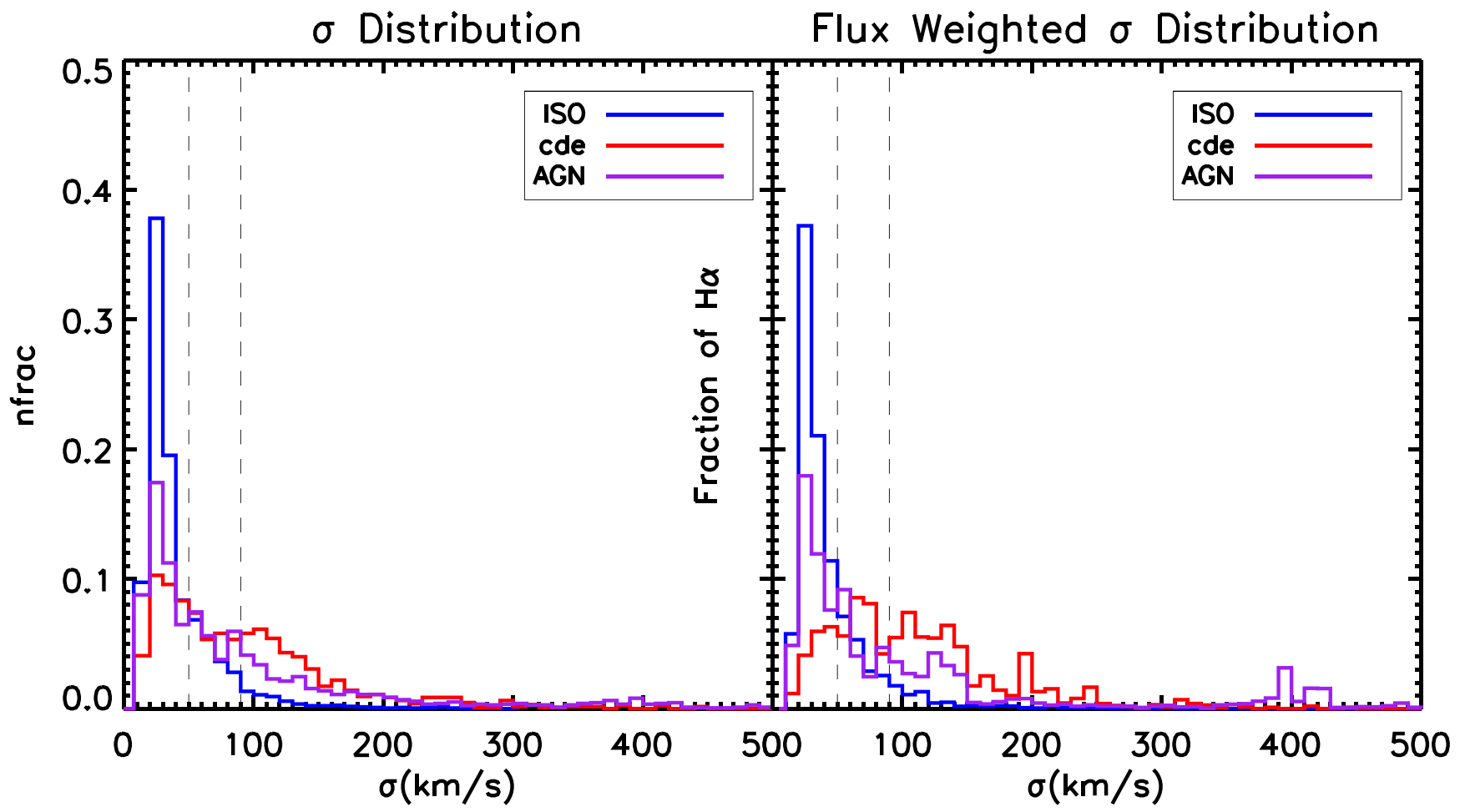

Figure 7. Line ratio vs. $\sigma$ as in Figure 6, but for isolated galaxies, coalesced mergers, and the four AGNs. Note that this figure is extended to a higher value of $\sigma$ than Figure 6.

star formation seen in strongly starbursting systems (e.g., Green et al. 2010). The highest observed velocity dispersions, above $90 \mathrm{~km} \mathrm{~s}^{-1}$, are caused by even more turbulent activity in the ISM of U/LIRGs. The most likely culprit is shocks caused by merger-driven gas flows, such as galactic winds (e.g.,
Armus et al. 1989, 1990; Rich et al. 2010; Sharp \& BlandHawthorn 2010). These lower-velocity shocks exhibit velocity dispersions consistent with the outflow velocity and the observed high- $\sigma$ gas, on the order of $100-200 \mathrm{~km} \mathrm{~s}^{-1}$ (Rich et al. 2010, 2011). The high- $\sigma$ component observed in 


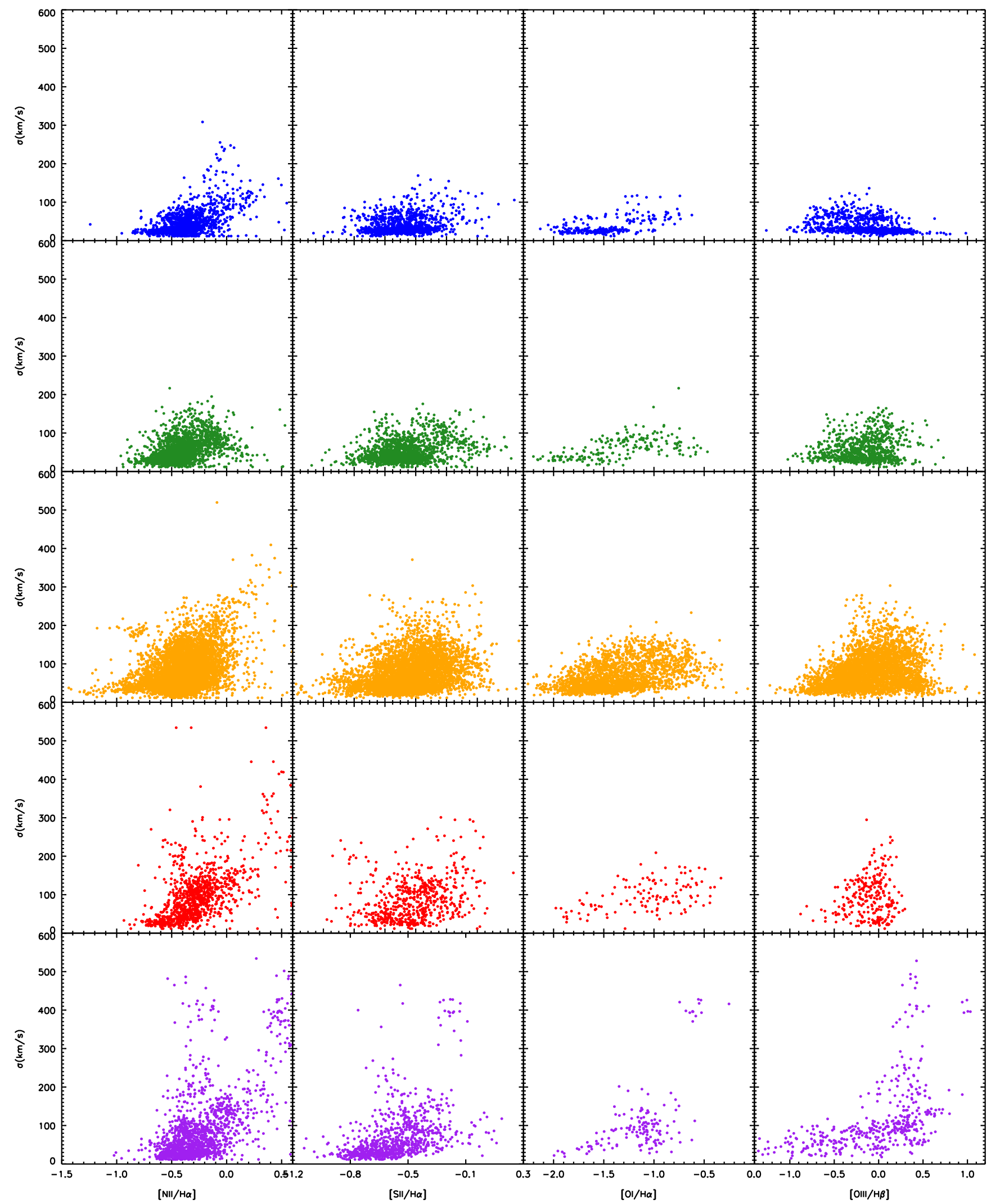

Figure 8. Line ratio vs. $\sigma$ as in Figure 4, for each merger stage, with "iso, a, b, cde" and AGNs represented by blue, green, orange, red, and purple from top to bottom, respectively.

our systems is also correlated with elevated emission-line ratios, as seen by Monreal-Ibero et al. (2010) and consistent with the radiative spectra of gas ionized by shocks. This combined elevation in emission-line ratios and velocity dispersions increases as a function of merger stage, and in the seven latest-merger-stage systems, there appears to be a 


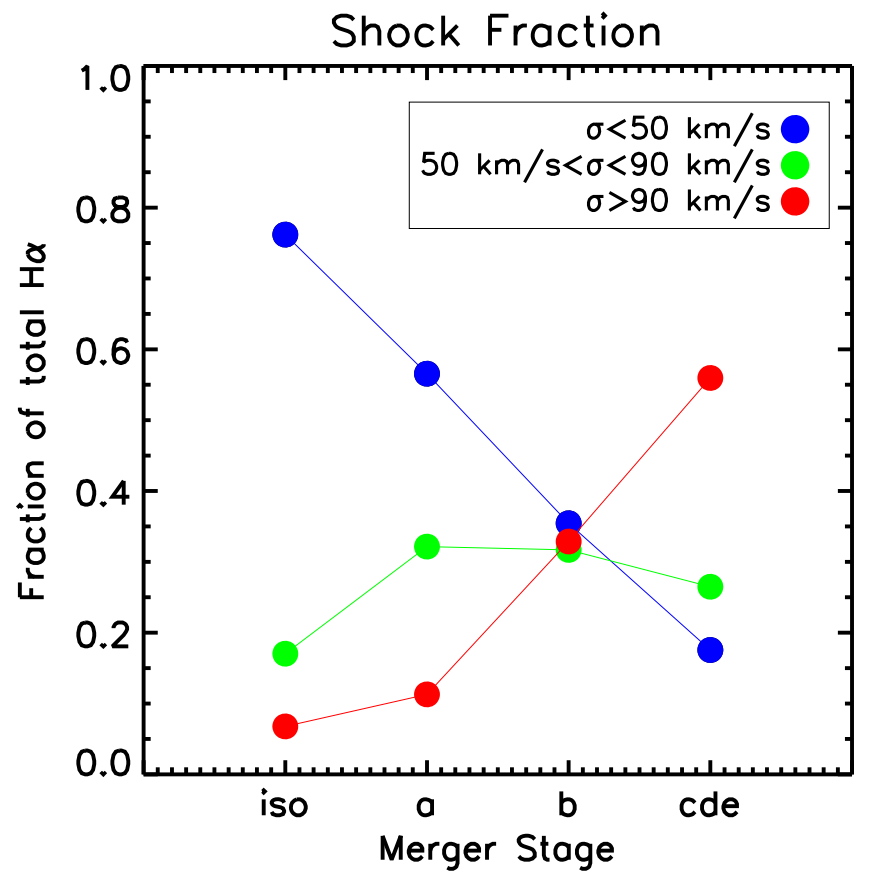

Figure 9. Here the contribution from shocks is traced by the overall velocity dispersion distribution. In each merger stage bin, the total fraction of $\mathrm{H} \alpha$ flux within each velocity dispersion range is calculated. Cutoffs of $\sigma=50$ and $90 \mathrm{~km} \mathrm{~s}^{-1}$ are chosen to distinguish between $\mathrm{H}$ II region velocity dispersions, more turbulent dispersions, and shock-dominated dispersions. These values are consistent with the overall distribution of spaxels seen in Figure 6.

significant contribution to the optical spectra from mergerinduced shocks.

There is the possibility of contribution from a less luminous AGN (classical LINER), which can similarly increase observed emission-line ratios and velocity dispersions. The spatial and spectrally resolved information provided by our IFS observations, however, shows not only elevated $\sigma$ and line ratios in the nuclear regions, but at many kiloparsecs from the nucleus, more consistent with widespread shocks (see Appendix C). We discuss further possible contamination from AGNs in Section 7.2.

\subsection{AGN Diagnostics and Contamination}

The conclusion that the composite spectra we observe are due to shocks assumes that there is no appreciable contribution to the optical spectra from an AGN. Although we have excluded the four AGN-dominated galaxies from our merger stage discussion, there remains the question of any low-level AGN contribution to the optical spectra in some of our systems. This is of somewhat more of a concern in the latest-stage mergers in our sample, as the optical emission is concentrated primarily in the nuclear regions. Although none of the galaxies in the merger sample have detectable $[\mathrm{Nev}]$ emission in the mid-IR, this does not necessarily rule out the possibility of a lower-luminosity AGN (Petric et al. 2011). Further, not all of the galaxies in our sample have been observed with Chandra, and some systems that have been observed with Chandra but show X-ray emission consistent with intense star formation may still harbor compton-thick AGNs (Iwasawa et al. 2009, 2011).

Another measure of AGN activity that can be derived from the mid-IR data is $6.2 \mu \mathrm{m}$ polycyclic aromatic hydrocarbon
(PAH) equivalent width (EW). In AGN-dominated spectra the hot dust continuum depresses the $6.2 \mu \mathrm{m}$ EW and the PAHs that generate the feature are apparently destroyed in the EUV photon field (Genzel et al. 1998; Lutz et al. 1999; Rigopoulou et al. 1999; Sturm et al. 2000; Armus et al. 2007; Desai et al. 2007; Wu et al. 2009; Fu et al. 2010; Petric et al. 2011). Stierwalt et al. (2013) determined $6.2 \mu \mathrm{m}$ PAH EW values from nuclear spectra for the entire GOALS sample, setting a value EW $>0.54 \mu \mathrm{m}$ for galaxies that are dominated by starbursts and $\mathrm{EW}<0.27$ for galaxies dominated by an AGN. Galaxies with EW values that fall between these values are considered composite systems.

To make a more conservative estimate of the shock fraction in the late-stage mergers, galaxies with $6.2 \mu \mathrm{m}$ PAH EW $<0.54 \mu \mathrm{m}$ are removed from the "cde" bin (these include IRAS 08355-4944, F10038-3338, F17207-0014, and F205514250 , with $6.2 \mu \mathrm{m}$ PAH EW of $0.19,0.03,0.31$, and $0.10 \mu \mathrm{m}$, respectively). This leaves three coalesced mergers, IRAS F12592+0436, F17138-1017, and F22467-4906, which have EW of $0.55,0.68$, and $0.45 \mu \mathrm{m}$, respectively. The total number of components with $\mathrm{H} \alpha$ above the $\mathrm{S} / \mathrm{N}$ cutoff in these three galaxies is $\sim 490$. The new velocity dispersion distribution and shock fraction are shown in Figures 10 and 11. Interestingly, when the potential low-luminosity AGN-bearing galaxies are excluded, the fraction of emission from broad, slow-shockdominated velocity dispersion components goes up, moving from roughly $55 \%$ to nearly $70 \%$ of the observed $\mathrm{H} \alpha$ flux. In Figure 10 IRAS F17138-1017 is classified as a composite system, while F12592+0436 and F22467-4906 host extended LINER-like emission, though in the complete absence of any indication of AGN activity these three systems are clear examples of shock-driven LIER hosts, with LIER emission contaminating and in some cases dominating the observable optical emission line gas.

\subsection{Prevalence of Shocks and Energy Budget}

Our results indicate an overall increase in the total contribution from shocks to the global optical spectra of middle- and late-stage merging U/LIRGs. The U/LIRGs in the WiFeS GOALS sample contain buried star formation that is so extinguished as to be unobservable at optical wavelengths, which may account in part for the relative increase from non$\mathrm{H}$ II region emission to the total observed emission-line flux.

A prime example of this is IRAS F01053-1746, which has an extended shock-like region in the optical co-spatial with a strong IR source indicative of buried star formation (Howell et al. 2010; Rich et al. 2011). Indeed, as noted in Rich et al. (2011), the total luminosity of the shocks in IRAS F010531746 is a negligible fraction of the bolometric luminosity, though they can still act as a means of removing energy from the infalling gas over the timescale of the merger process.

Regardless, the observable optical emission in our sample shows a combination of $\mathrm{H}$ II region emission and ionization by slow shocks, increasing as a function of merger stage. This combination manifests in some systems as a "composite" spectrum, though in this case "composite" means starburst + shocks rather than starburst+AGNs. Indeed, in Rich et al. (2014) we showed that when only nuclear spectra are considered, $75 \%$ of the "composite" galaxies in our sample are composite simply as a result of a sizable contribution from shocks to their emission-line spectra. 


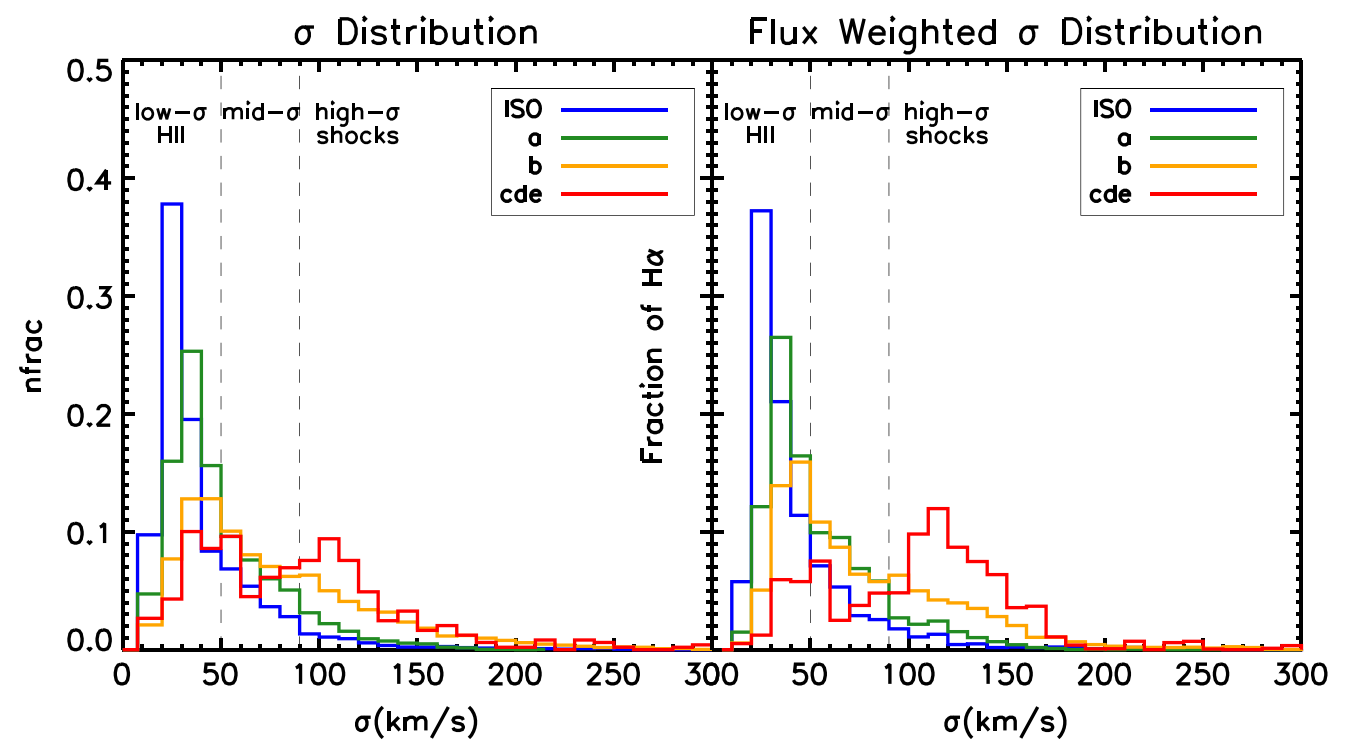

Figure 10. Reproduction of Figure 7 with low $6.2 \mu \mathrm{m}$ PAH EW galaxies excluded from "cde. " In this case, only three galaxies remain in the "cde" bin, IRAS F12592 +0436, F17138-1017, and F22467-4906.

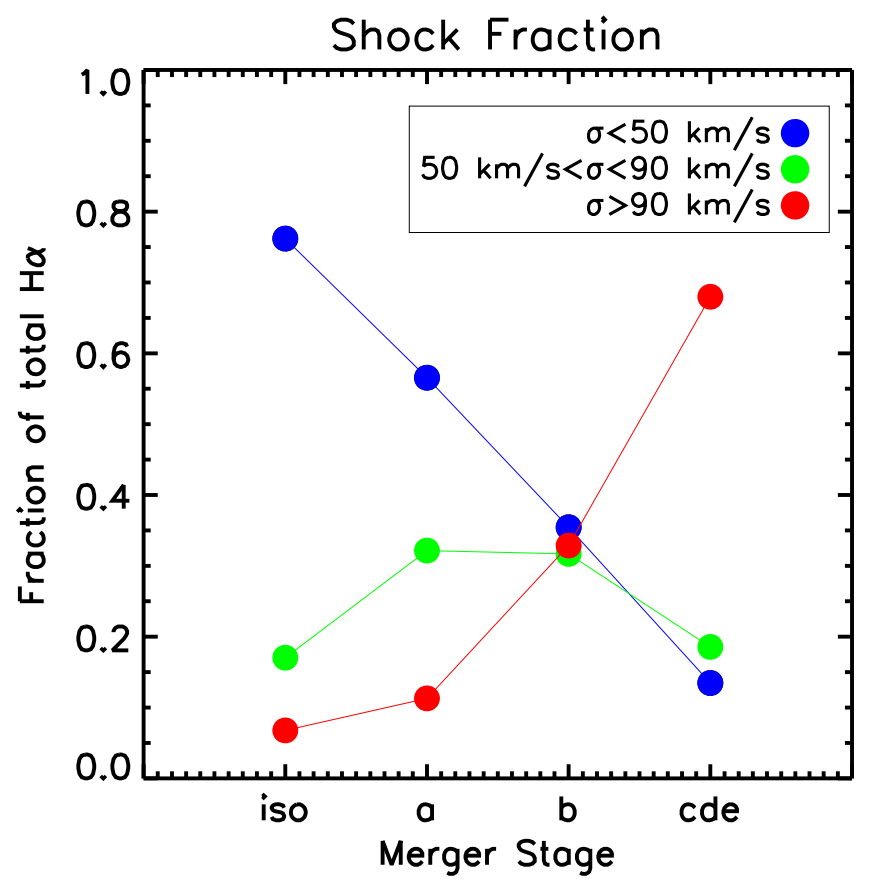

Figure 11. Reproduction of Figure 9 with low $6.2 \mu \mathrm{m}$ PAH EW galaxies excluded from "cde," as in Figure 12.

\section{SUMMARY}

We have analyzed WiFeS optical integral field spectroscopic data cubes of 27 systems from the GOALS sample, 23 of which are a combination of at least two interacting galaxies, ranging from widely separated pairs approaching first pericenter to coalesced systems on their way to becoming elliptical merger remnants. Our analysis focuses on continuum-subtracted emission-line gas, in particular through a decomposition of individual emission-line profiles into one, two, or three distinct kinematic components. We use the results of our emission-line fits to create line ratio maps, line diagnostic diagrams, and velocity dispersion distributions for each individual galaxy observed. Maps and plots of the results for every system are presented in Figures 12-16 and details of individual systems are provided in the Appendix. We consider $\mathrm{H} \alpha$-flux-weighted line component velocity dispersion distributions to avoid overweighting regions of low-surface-brightness emission. The results from each galaxy are placed into bins according to merger stage, and the evolutions of the emission-line ratios and velocity dispersions are tracked as a function of merger progress. Our main conclusions are as follows.

1. Isolated systems and wide pairs are dominated primarily by $\mathrm{H}$ II region emission $(\sim 75 \%$ and $\sim 55 \%$ of $\mathrm{H} \alpha$ emission, respectively), with line profiles primarily with $\sigma$ of a few tens of kilometers per second.

2. All interacting systems exhibit emission with slightly higher velocity dispersions $\left(50<\sigma<90 \mathrm{~km} \mathrm{~s}^{-1}\right)$, likely associated with turbulent star formation (e.g., Genzel et al. 2008; Green et al. 2010). This component makes up roughly $30 \%$ of the total observed $\mathrm{H} \alpha$ flux throughout the merger process.

3. Closely interacting pairs and coalesced mergers show a strong $\sigma$ component above $100 \mathrm{~km} \mathrm{~s}^{-1}$ and an increasingly dominant contribution from composite and LINERlike emission-line ratios. In the absence of an AGN, we assume that the broadened $\sigma$ component above $90 \mathrm{~km} \mathrm{~s}^{-1}$ is tracing an increasing fractional contribution from shocks, passing $50 \%$ of the total observed $\mathrm{H} \alpha$ flux in the coalesced mergers.

We consider the IFS data of four AGN-dominated systems in our sample separately. The AGN-dominant systems are detected in $[\mathrm{Ne} v]$ in the mid-IR and show AGN-like X-ray colors and spectra in Chandra and XMM-Newton data (Franceschini et al. 2003; Iwasawa et al. 2011; Petric et al. 2011). We consider the role that less dominant AGNs may play in the latest-stage mergers in our system by using the mid-IR diagnostic $6.2 \mu \mathrm{m}$ PAH EW as a proxy for AGN activity. Our conclusions are as follows.

1. The four AGNs show a significant component at $\sigma$ of $300-500 \mathrm{~km} \mathrm{~s}^{-1}$ and emission-line ratios clearly 


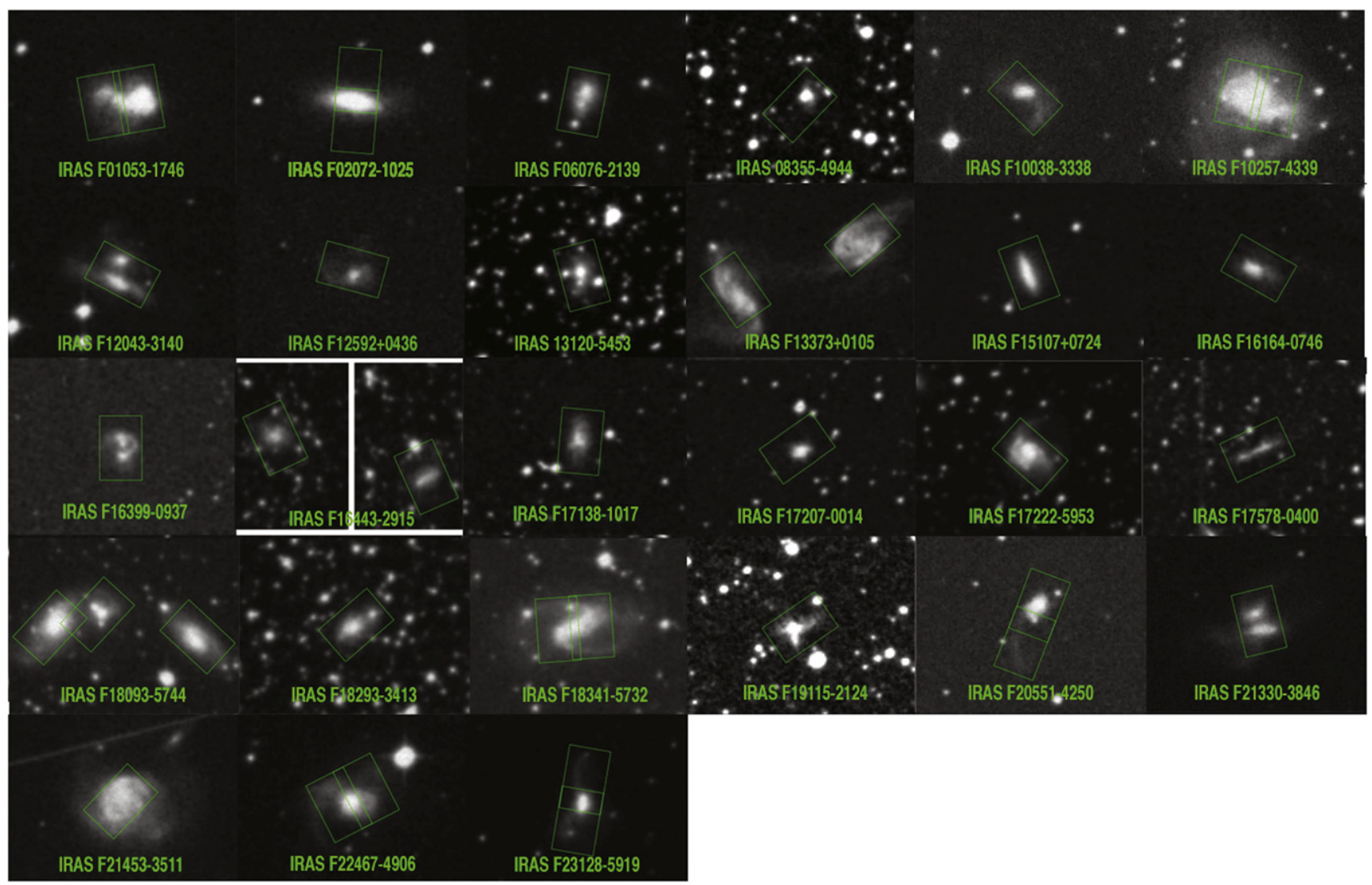

Figure 12. DSS $R$-band images with individual WiFeS pointings overlaid in green. Coordinates for the pointings are given in Table 2.

approaching and entering the Seyfert region of the emission-line diagnostic diagrams. The AGNs also contain shock-like velocity dispersions, driven by either the AGN itself, galactic winds (observed in blueshifted Na D absorption), or merger-induced gas flows.

2. When the low $6.2 \mu \mathrm{mEW}$ coalesced mergers are removed from the non-AGN sample, the shock-like high- $\sigma$ component increases to $70 \%$ of the observed $\mathrm{H} \alpha$ emission in the remaining three systems. Stierwalt et al. (2013) note that some galaxies show strong evidence for enhanced $\mathrm{H}_{2}$ emission due to starburstdriven outflows and shocks rather than AGNs, with correspondingly low PAH $6.2 \mu \mathrm{m} \mathrm{EW}$, lending further ambiguity to the picture.

Spatially resolved spectroscopy is clearly a powerful tool for distinguishing non-AGN composite emission, particularly in systems where widespread shocked ISM is expected as a result of a powerful starburst or an ongoing gas-rich interaction. Recent multiwavelength studies of systems from the GOALS sample and elsewhere have shown promising routes to further study the impact that shocks have on the environment of $\mathrm{U} /$ LIRGs (Mazzarella et al. 2012; Inami et al. 2013; U et al. 2013). New surveys of hundreds and thousands of galaxies with IFU spectroscopy, namely, CALIFA, SAMI, and MaNGA (Sánchez et al. 2012; Croom et al. 2012; Bundy et al. 2015), are revealing a complex mix of forces at work in less luminous galaxies, including LINER excitation in nonmergers caused by shocks, as well as a warm ionized ISM heated by old stars and UV photons that escape H II regions (Arribas et al. 2014; Ho et al. 2014; Belfiore et al. 2015). The combination of complementary, resolved multiwavelength data that probe the multiple sources of activity will be the key to understanding the complex interplay between star formation, shocks, AGNs, and the ISM in galaxies throughout the universe.

The authors acknowledge ARC support under Discovery project DP0984657. This research has made use of the NASA/ IPAC Extragalactic Database (NED), which is operated by the Jet Propulsion Laboratory, California Institute of Technology, under contract with the National Aeronautics and Space Administration. This research has also made use of NASA's Astrophysics Data System and of SAOImage DS9 (Joye \& Mandel 2003), developed by the Smithsonian Astrophysical Observatory.

\section{APPENDIX A \\ NOTES ON INDIVIDUAL SYSTEMS AND MAPS}

This appendix contains brief notes about each system from our WiFeS GOALS sample. The reader may find it useful to refer to Figures 12-16, which include DSS R-band or $H S T$ ACS I-band images matching the field of view of the WiFeS GOALS observations combined with emission-line ratio maps, emission-line ratio diagnostic diagrams, velocity dispersion distribution histograms, velocity dispersion versus emissionline ratio diagrams, and maps of the distribution of low-, mid-, and high-s velocity components. Some of the notes on individual systems are adapted from those systems already published in Rich et al. (2012).

IRAS F01053-1746 (IC 1623). This system contains two very closely interacting spiral galaxies. It is kinematically very 

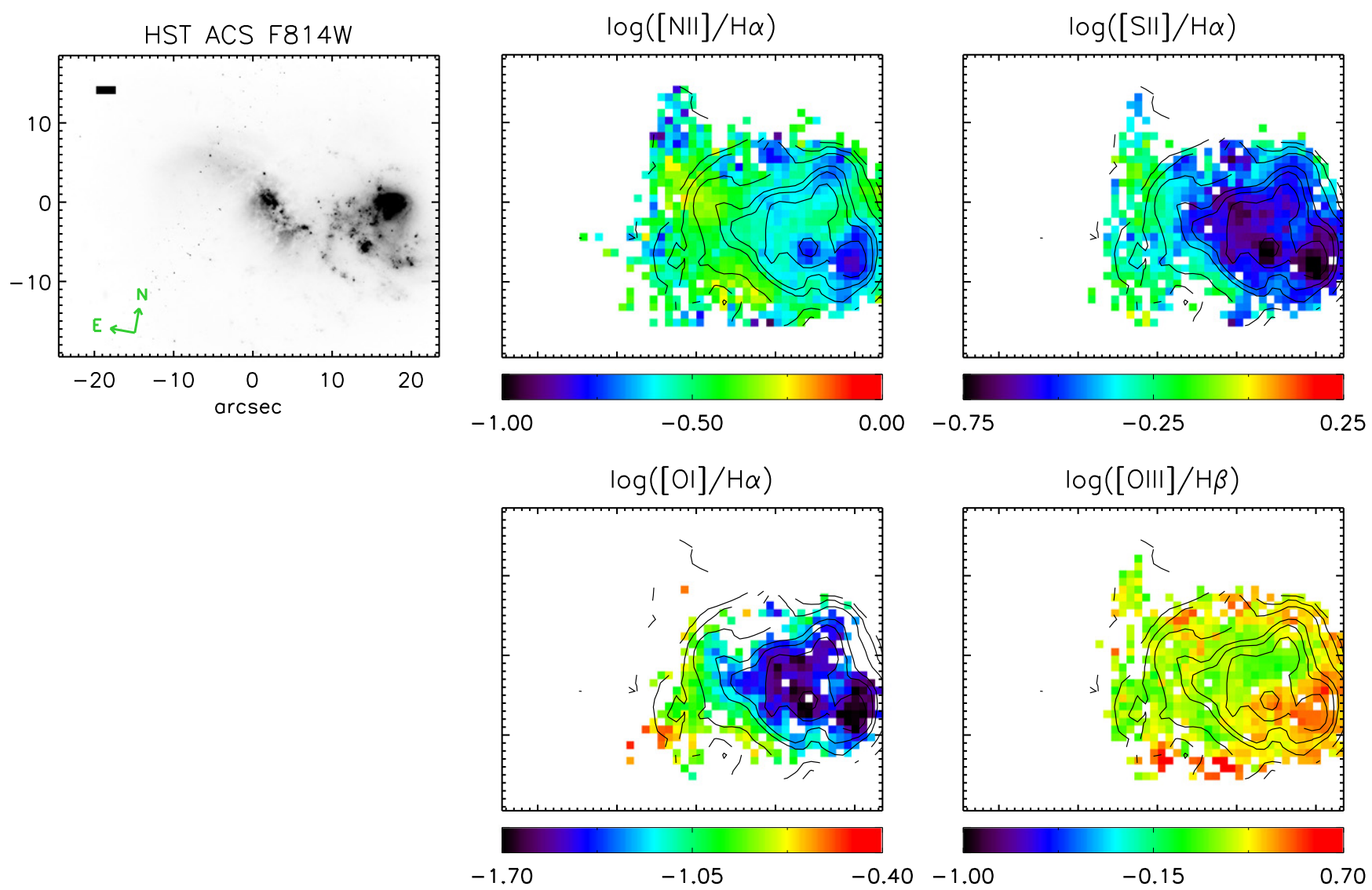

Figure 13. The top left panel shows an HST I-band image or a DSS R-band image aligned with the total WiFeS field of view with a 1-kpc bar. The four panels to the right show emission line ratio maps for $[\mathrm{N}$ II $] / \mathrm{H} \alpha,\left[\mathrm{S}_{\mathrm{II}}\right] / \mathrm{H} \alpha,\left[\mathrm{O}_{\mathrm{I}}\right] / \mathrm{H} \alpha$, and $[\mathrm{O} \mathrm{III}] / \mathrm{H} \beta$ with $\mathrm{H} \alpha$ contours measured from our data overlaid.

(The complete figure set (31 images) is available.)

complex and exhibits evidence of widespread radiative shocks (Rich et al. 2011). The eastern system is very intensely starforming, as seen in the infrared (e.g., Howell et al. 2010), but is so enshrouded in dust that our optical spectra do not trace any of the buried star formation.

IRAS F02072-1025 (NGC 839). This system is described in great detail in Rich et al. (2010). It is a nearly edge-on starburst galaxy in a compact group (HCG 16). Our emission-line maps trace the shocks associated with the starburst-driven outflow, and we see evidence of a post-starburst $\mathrm{E}+\mathrm{A}$ spectrum in portions of the galaxy.

IRAS F06076-2139. Arribas et al. (2008) describe this ULIRG in great detail in their IFS study of ULIRGs. They determined that the system consists of two galaxies that may never merge given their significantly different velocities, though they are interacting as evidenced by a ring of $\mathrm{H} \alpha$ emission (Elmegreen \& Elmegreen 2006; Arribas et al. 2008). Our velocity dispersion and line ratio data imply that the northern galaxy is dominated by star formation, with some amount of composite ratios and $\sigma$ in the southern galaxy. The southern galaxy in this closely interacting pair has a fairly low $6.2 \mu \mathrm{m}$ PAH EW, which also corresponds to enhanced [N II]/ $\mathrm{H} \alpha$ in our maps.

IRAS 08355-4944. While HST I-band images show remnant tidal tails extending nearly $20 \mathrm{kpc}$, only the central $5 \mathrm{kpc}$ or so appears to harbor the entirety of the intense ongoing star formation in this system. Our spectra are dominated by $\mathrm{H}$ II region emission, with evidence in some portions of the system of a blueshifted component with low-velocity shock-dominated line ratios that could be associated with a galactic wind. This is in agreement with the IFS observations of Monreal-Ibero et al. (2010).

IRAS F10038-3338 (ESO 374-IG032). This post-merger exhibits significant ongoing star formation in its southwestern tidal arm, unlike the two other coalesced systems in our sample. The total line emission in this region is much weaker than in the nucleus, inducing a large uncertainty in the extinction map and $\left[\mathrm{O}_{\mathrm{II}}\right]$ lines, creating the discrepant values seen in the metallicity gradients for this galaxy (Rich et al. 2012). This system hosts an $\mathrm{OH}$ megamaser and has soft $\mathrm{x}$-ray emission, all consistent with the advanced stage of merging and increasingly intense nuclear starburst (Henkel \& Wilson 1990; StaveleySmith et al. 1992; Darling \& Giovanelli 2002; Iwasawa et al. 2009). Our spectra also show evidence for extended offnuclear shock emission dominating in areas where there is little to no evidence of ongoing star formation, consistent with the IFU observations of Monreal-Ibero et al. (2010).

IRAS F10257-4339 (NGC 3256). This advanced merger is the nearest galaxy in our sample $(z \sim 0.0094)$ and is well studied. As with IRAS F01053-1746, one of the galaxies in IRAS F10257-4339 is very extinguished. The second, buried system and its nucleus are revealed at longer wavelengths, south of the main optical nucleus (Alonso-Herrero et al. 2002; Rothberg \& Fischer 2010). The gas and tidal tails in this system extend tens of kiloparsecs from the nuclear regions (e.g., Rothberg \& Fischer 2010). Our IFU mosaic covers only the central $6 \mathrm{kpc}$, though this appears to be the physical extent of 

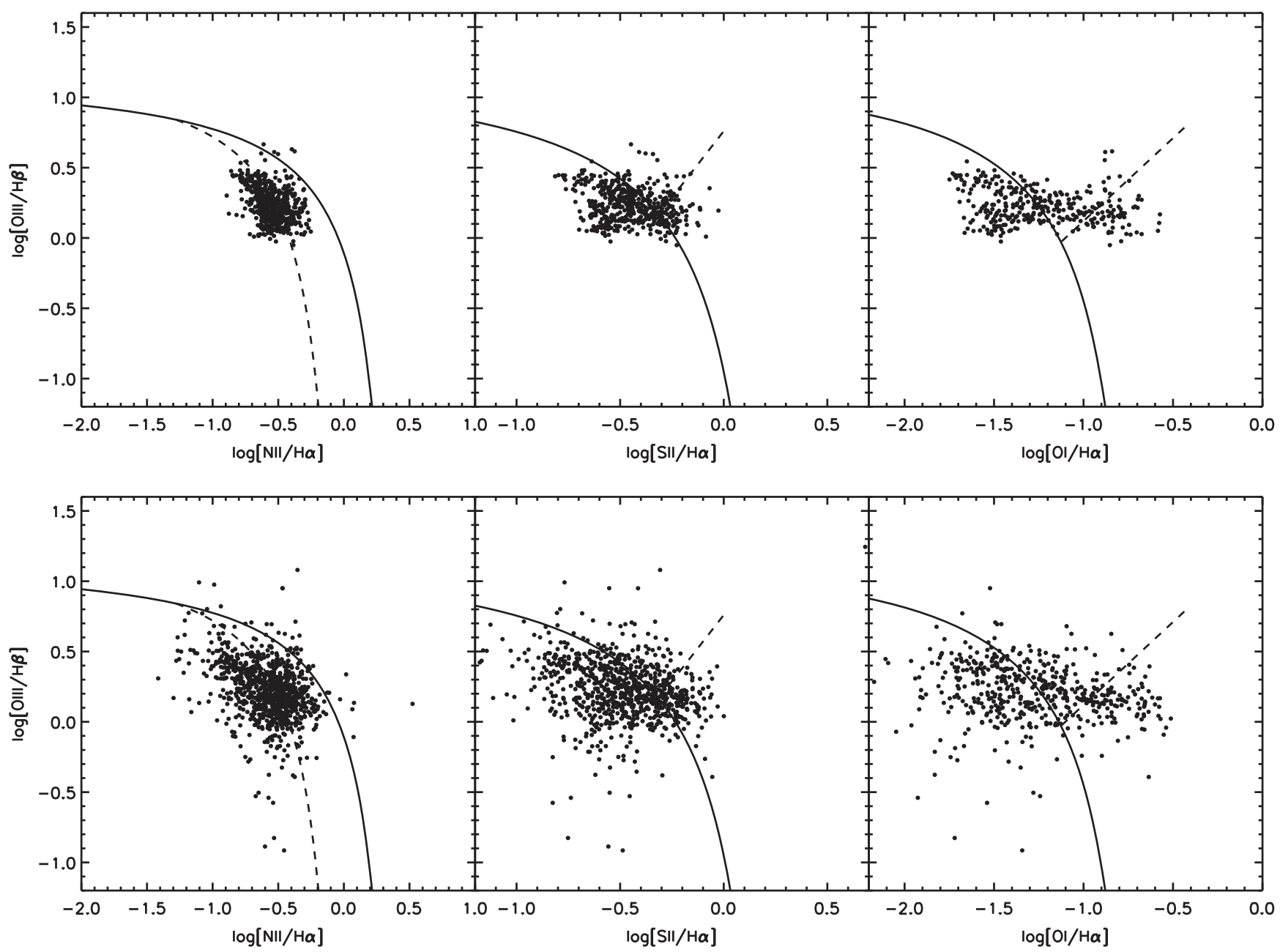

Figure 14. The six panels are standard diagnostic diagrams (Kewley et al. 2006). Points in the top three correspond to the total flux ratios in a single spaxel. Points in the bottom three correspond to line ratios from the individual components above $\mathrm{S} / \mathrm{N}$ of 3 in each line. The same diagrams for the remaining portion of the sample are in the figure Set.

(The complete figure set (31 images) is available.)

most of the ongoing star formation in this system. NGC 3256 also shows evidence for widespread shocks in our data and is discussed alongside IC 1623 in Rich et al. (2011).

IRAS F12043-3140 (ESO 440-IG058). This pair of closely interacting galaxies is poorly studied, and there exists no higher spatial resolution imagery to examine. The DSS image, though, shows tidal features indicative of the ongoing interaction. Our IFS data indicate a combination of ongoing star formation and shocked gas, seen in the diagnostic diagrams as $\mathrm{H}$ II region + composite spaxels and with a tail out to $\sigma \sim 200 \mathrm{~km} \mathrm{~s}^{-1}$ in the velocity dispersion distribution. Our map shows that the enhanced ratios seem to form a cone extending to the north and south of the southern galaxy, and agrees with the IFU observation of Monreal-Ibero et al. (2010). The overlap in the two galaxies and the lower $\mathrm{S} / \mathrm{N}$ of our data make it difficult to discern whether this is indeed associated with an outflow from the southern galaxy.

IRAS F12592+0436 (CGCG 043-099). This late-stage merger is very dusty, and subsequently the blue data have mostly too low $\mathrm{S} / \mathrm{N}$ to measure any emission in single spaxels. The red data indicate low line ratio and $\sigma$ gas in a tidal tail indicative of star formation and higher line ratio and $\sigma$ data toward the nucleus. Our nuclear spectrum indicates composite behavior dominated by shocks. Poggianti \& Wu (2000) found balmer emission and absorption indicative of star formation, with no indication of Seyfert behavior. X-ray and IR data indicate a galaxy with ongoing star formation and no indication of an AGN, consistent with our results (Rush et al. 1996; Petric et al. 2011; Stierwalt et al. 2013).

IRAS 13120-5453. This nearby ULIRG is dominated by AGN emission at all wavelengths. X-ray data indicate an AGN signature (Iwasawa et al. 2009, 2011), and mid-IR PAH values and $[\mathrm{Ne} \mathrm{v}$ ] are consistent with a galaxy dominated by an AGN (Farrah et al. 2007; Pereira-Santaella et al. 2010; Petric et al. 2011). Our optical IFS data are quite consistent with this picture: the strongest emission we detect is dominated by Seyfert-like ratios in the diagnostic diagrams, and the velocity dispersion distribution shows a contribution from a high- $\sigma$ component in the nuclear regions of $300-400 \mathrm{~km} \mathrm{~s}^{-1}$ in addition to the lower $\sim 100 \mathrm{~km} \mathrm{~s}^{-1}$ component.

IRAS F13373+0105 W (NGC 5257). Although NGC 5257 and its equal-mass partner NGC 5258 are still widely separated and retain much of their structure, they exhibit interaction features including tidal tails and a bridge between the two galaxies. The spiral arms show regions of strong star formation with some signatures of post-starburst populations away from 


\section{IRAS F01053-1746}

$\sigma$ Distribution

Flux Weighted $\sigma$ Distribution
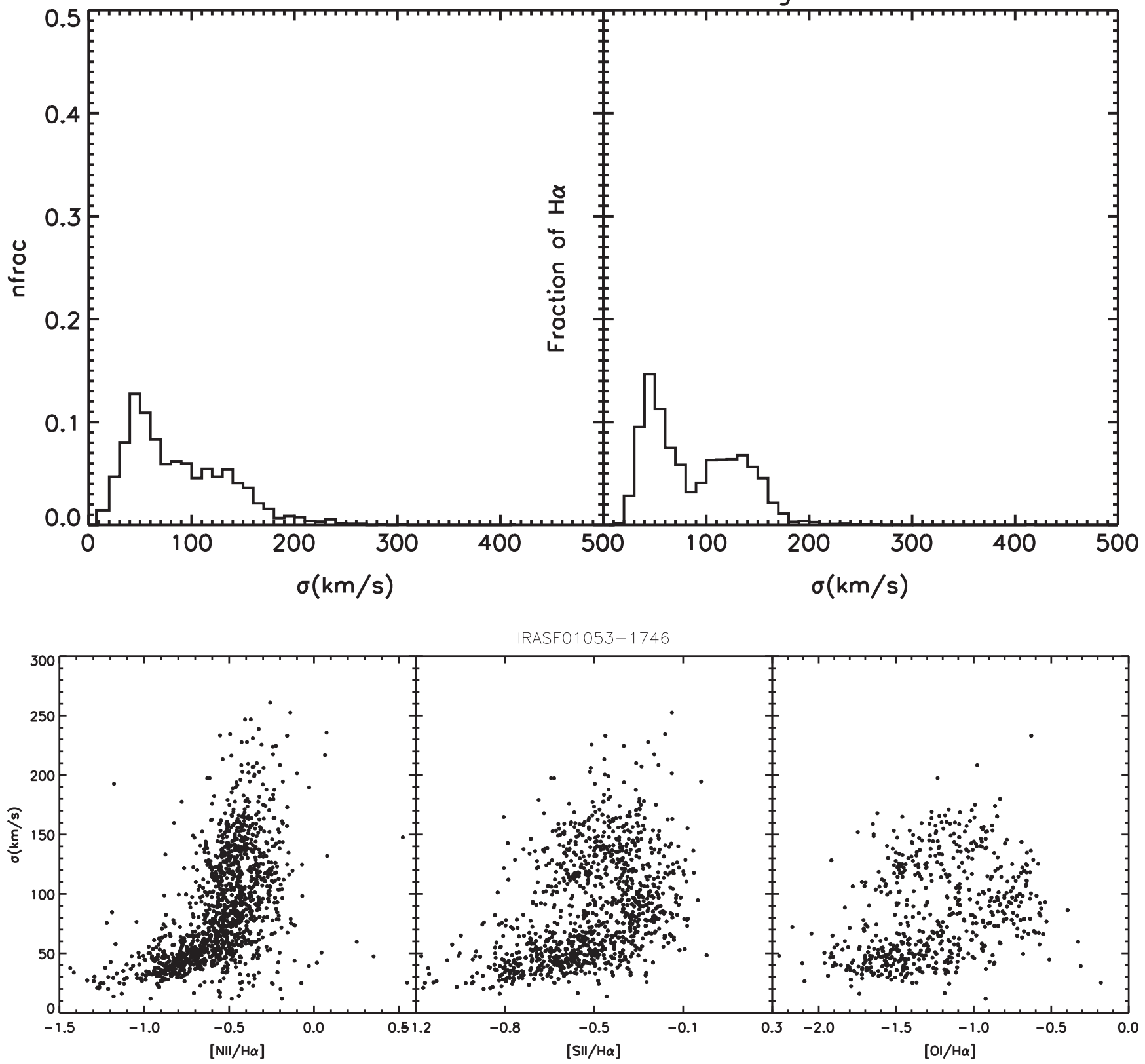

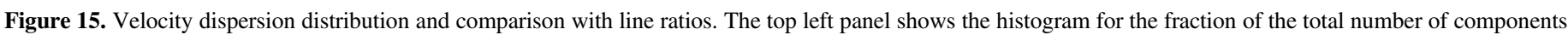

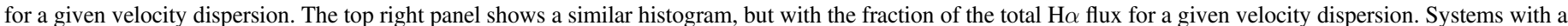

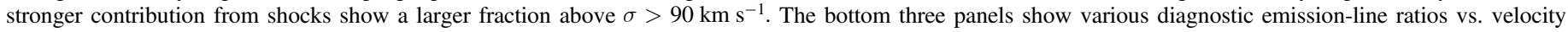

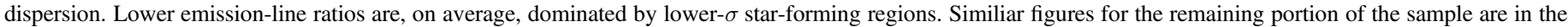
figure Set.

(The complete figure set (31 images) is available.)

the nucleus. The nuclear regions of NGC 5257 are dominated by an older stellar population as the intense nuclear starburst associated with the later stages of major mergers has not yet begun. The extinction is higher in the nuclear regions, and the measurable line ratios place the nucleus in the composite region of the standard diagnostic diagrams, indicating possible LINER activity-previous nuclear observations and integrated spectrophotometry are consistent with the higher nuclear extinction and overall line ratios we observe (Veilleux et al. 1995; Kewley et al. 2001b; Moustakas \& Kennicutt 2006).
IRAS F13373+0105 $E$ (NGC 5258). NGC 5258 is a near twin to NGC 5257 in mass and luminosity. Our spectra indicate higher extinction and a flatter gradient, though the extent of measurable $\mathrm{H}$ in region metallicities is smaller than in NGC 5257. Again similar to NGC 5257, the nuclear region of NGC 5258 is dominated by older stars and very little line emission, and there is some evidence of younger poststarburst populations away from the nucleus. The strongest line emission is associated with the knot of star formation to the southwest of the nuclear region. 

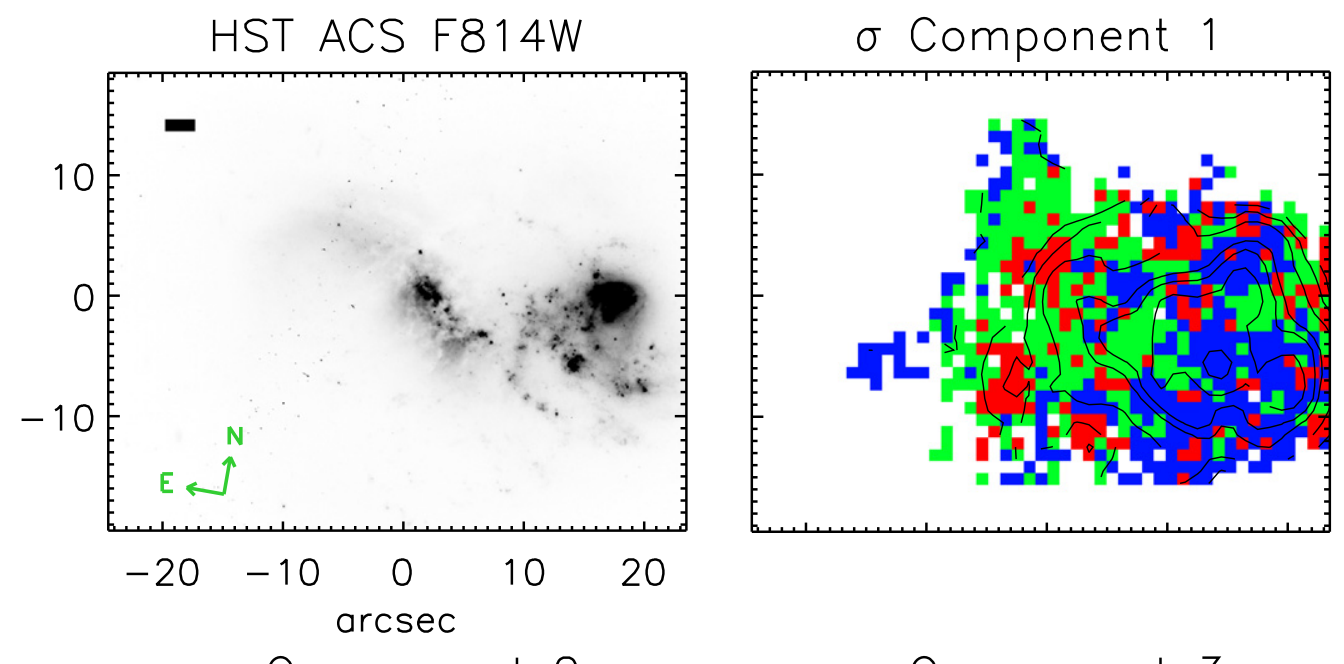

$\sigma$ Component 2
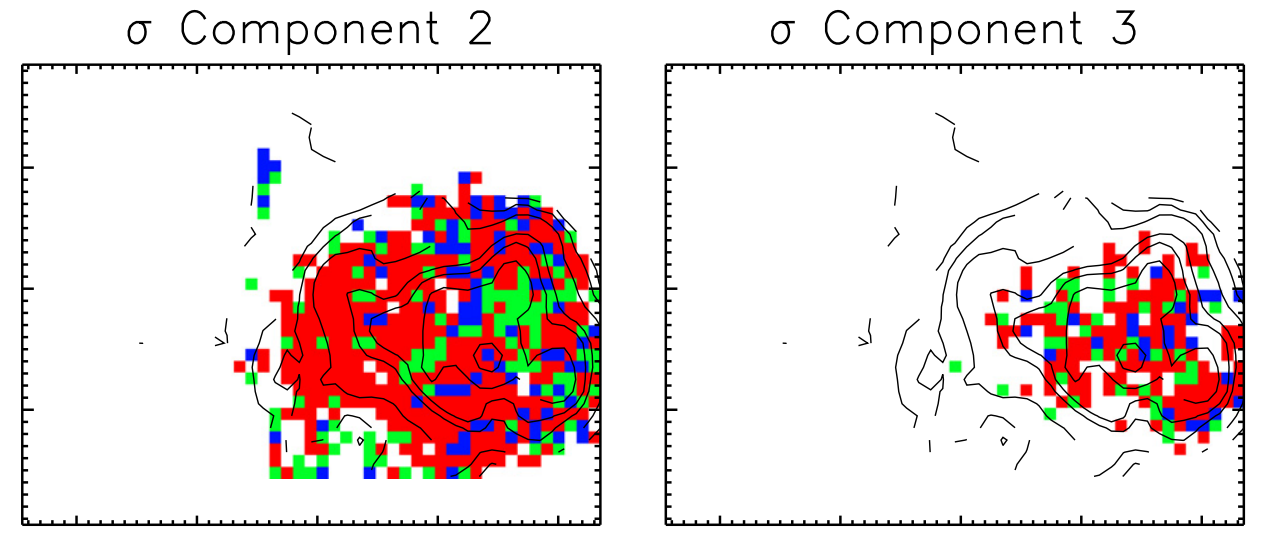

Figure 16. Maps of each velocity component color coded by velocity dispersion classification with cutoffs as shown in Figures 5 and 6 and discussed in Section 7 : blue, low- $\sigma\left(\sigma<50 \mathrm{~km} \mathrm{~s}^{-1}\right)$, green mid- $\sigma\left(50 \mathrm{~km} \mathrm{~s}^{-1}<\sigma<90 \mathrm{~km} \mathrm{~s}^{-1}\right)$ and high- $\sigma\left(\sigma>90 \mathrm{~km} \mathrm{~s}^{-1}\right)$ velocity dispersion classification. IRAS F01053-1746, for instance shows a strong combination of $\mathrm{H}$ II region and turbulent star formation, seen as blue and green components as well as a widespread high- $\sigma$ component from shocks. Many of the mid to late stage mergers show a similar pattern. The upper left panel shows the galaxy image for comparison and the maps are shown with H $\alpha$ contours overlaid, as with the figures in Appendix A.

(The complete figure set (31 images) is available.)

IRAS F15107+0724 (CGCG 049-057). This galaxy bears a low-luminosity OH megamaser (Martin et al. 1988) and was classified as an $\mathrm{H}_{\text {II }}$ region galaxy by Kim et al. (1995) and Veilleux et al. (1995). Our IFS data are dominated primarily by an older stellar population in the blue, with the emission lines primarily indicative of ongoing star formation plus some composite contribution, likely shocks given the velocity dispersion distribution and relatively high $6.2 \mu \mathrm{m}$ PAH EW value of $0.51 \mu \mathrm{m}$ (Stierwalt et al. 2013). An HST NICMOS $H$-band image provides the highest-resolution data available, showing what appears to be a relatively undisturbed spiral disk (Haan et al. 2011). This is in keeping with its status as an isolated galaxy (Yuan et al. 2010).

IRAS F16164-0746. This coalesced merger is AGN dominated in the X-ray and mid-IR (Iwasawa et al. 2009, 2011; Petric et al. 2011). Our optical spectra show rotation and a narrow and a broad component, consistent with star formation and shocks, though our line ratio map shows a composite spectrum. Our data do not have sufficient $\mathrm{S} / \mathrm{N}$ to detect $[\mathrm{O}$ III]/ $\mathrm{H} \beta$ where the other line ratios increase toward the nucleus, where more LINER-like ratios dominate (Veilleux et al. 1995). Our nuclear spectrum is somewhat ambiguous, indicating composite, starburst (SB), and Seyfert-like spectra with [O III]/ $\mathrm{H} \beta$ near unity.
IRAS F16399-0937. This close-merger pair shows $\mathrm{H}$ II region + composite emission and a velocity dispersion distribution dominated by star formation. The emission-line ratio map indicates a ring of star-forming gas surrounding the northern nucleus. The northern nucleus has much stronger emission-line ratios, though very few spaxels are detected in both $[\mathrm{O}$ III] and $\mathrm{H} \beta$. The composite nuclear spectrum of the northern nucleus has $[\mathrm{O} \mathrm{III}] / \mathrm{H} \beta$ near unity, indicating LINERlike emission. This may be associated with shocks, possibly caused by an outflow. There is some indication of broad blue wings in the $\mathrm{Na} \mathrm{D}$ profile possibly associated with such an outflow (e.g., Rupke et al. 2005), though no fit was performed.

IRAS F16443-2915 N and S (ESO 453-G005). This widely separated pair of galaxies appears to be completely dominated by star formation. The emission-line ratio maps are dominated by $\mathrm{H}$ II emission, and the velocity dispersions in both systems are primarily a few tens of kilometers per second. The southern galaxy does show a larger turbulent component approaching $\sigma \sim 100 \mathrm{~km} \mathrm{~s}^{-1}$, but neither indicates any shock component. These galaxies show no apparent signs of interaction, though the available imaging data are low resolution.

IRAS F17138-1017. Our observations of this coalesced merger are consistent with the IFS observations of MonrealIbero et al. (2010). The central regions of the galaxy are dominated by star formation, with an underlying shock-like 
velocity dispersion component. In the outer portions of the galaxy, our line ratios are also consistent with a dominant contribution from shocks in both line ratio and velocity dispersion. There is no indication of any AGN activity at any wavelength, with a very high $6.2 \mu \mathrm{m}$ PAH EW of $0.68 \mu \mathrm{m}$ (Stierwalt et al. 2013).

IRAS F17207-0014. This ULIRG is the second brightest in the GOALS sample, behind only MRK 231. Despite its high $L_{\mathrm{IR}}$, the X-ray data from Chandra and XMM-Newton are apparently dominated by the starburst (Franceschini et al. 2003; Iwasawa et al. 2011). The $6.2 \mu \mathrm{m}$ PAH EW is low $(0.3 \mu \mathrm{m})$, though there is no detected [Ne v] emission (Petric et al. 2011; Stierwalt et al. 2013). Arribas \& Colina (2003) carried out early IFS observations of this system and suggest that this ULIRG is not evolving into a QSO. Our data show composite-like line ratios,though the overall velocity dispersion distribution is quite high, with $\sigma$ exceeding $100 \mathrm{~km} \mathrm{~s}^{-1}$ in nearly all measurable spaxels. Rupke et al. (2005) detect blueshifted $\mathrm{Na} \mathrm{D}$ absorption consistent with a galactic wind, which we also see in our data. In a recent study, Medling et al. (2015) analyzed the nuclear region in great detail with AO-aided nearIR IFU data, which also show evidence of outflow-driven shocks. Taken all together, the data indicate an intense starburst with a galactic wind driving low-velocity shocks.

IRAS F17222-5953 (ESO 138-G027). This system is more akin to a typical, non-interacting, strongly starbursting spiral galaxy in our sample. It is in the vicinity of a few other galaxies, including the similarly bright ESO 138-G026, but even the nearest galaxy is at a projected distance of over $100 \mathrm{kpc}$, and IRAS F17222-5953 is not yet interacting with any of these systems. Our IFU data applied to the $[\mathrm{N}$ II] $/ \mathrm{H} \alpha \mathrm{v}$ [O III] $/ \mathrm{H} \beta$ BPT diagram show a clean curve following the shape of the Sloan Digital Sky Survey sequence of local starforming galaxies (e.g., Kewley et al. 2006), but with a slight apparent shift in total $[\mathrm{N} \mathrm{II}] / \mathrm{H} \alpha$. We interpret this shift as an overall nitrogen enhancement (Pérez-Montero \& Contini 2009).

IRAS 17578-0400. There is very little information available about this system in the literature. Our data have very low $\mathrm{S} / \mathrm{N}$ in the blue, though the binned nuclear spectrum is indicative of pure star formation. This is consistent with the [N II] and [S II]/ $\mathrm{H} \alpha$ line ratios and the velocity dispersion distribution, which shows little to no indication of any appreciable non-H II region component and a rotation-dominated velocity field.

IRAS F18093-5744 N (IC 4687). We classify this galaxy as a close merger; in fact, it is a member of a triplet. IC 4687 is undergoing a close merger with the less massive starburst IC 4686, classed as a Wolf-Rayet galaxy by Kovo \& Contini (1999) and Fernandes et al. (2004). IC 4687 itself is also in a wide merger with the equally massive spiral IC 4689. The archived HST images of IC 4687 show a complex morphology tangled up with IC 4686: gas and dust from IC 4687 appear to be obscuring the less massive system. Our IFU data cover the entirety of IC 4686/4687, and the metallicities we measure are consistent with the expected metallicties in the outskirts of IC 4687 as extrapolated from the gradient we present in this paper, as well as a low-metallicity, flattened gradient in IC 4686. The kinematic information from our data also indicates that we are indeed seeing gas from both systems.

IRAS F18093-5744 S (IC 4689). This spiral galaxy is slightly less massive and luminous than IC 4687 and less well observed, though it is still intensely star-forming (Howell et al. 2010). It is less morphologically disturbed than the other two interacting galaxies IC 4686/4687, though its gradient is quite flattened already according to our observations (Rich et al. 2012). Although it is widely separated from IC 4687, we include it as part of the closely interacting system of IRAS F18093-5744.

IRAS F18093-5744 C (IC 4686). IC 4686 is a compact strongly starbursting galaxy with Wolf-Rayet features in its spectrum (Kovo \& Contini 1999; Fernandes et al. 2004). As noted in Rich et al. (2012), this galaxy shows a strong moderate $\sigma$ component with lower line ratios. This may be due to the very compact nature, causing an overlap in the kinematics that may require a higher-component line fit or higher-resolution spectroscopy than have been considered in this thesis.

IRAS F18293-3413. The IR-bright source in this galaxy is the northern, highly extinguished galaxy. $K$-band data from NIRC and HST NICMOS show very buried spiral structure (Väisänen et al. 2008a; Haan et al. 2011). The southern galaxy appears elliptical, with our IFS spectra having no detectable emission-line gas and a continuum indicative of an old stellar population. The northern galaxy is dominated by star formation with some contribution from shocks in the outskirts of the galaxy. The study of Chandra observations by Iwasawa et al. (2009, 2011) shows resolved soft X-ray emission possibly associated with an outflow, but with no indication of an AGN.

IRAS F18341-5732 (IC 4734). Like IRAS F17222-5953, this isolated galaxy is in the vicinity of a few other luminous galaxies, but is not yet undergoing any interactions. Our nuclear spectra are dominated by a LINER combined with an aging stellar population. The strongest sites of star formation are where the bar in this galaxy meets the spiral arms, evidenced by the two strong clumps of $\mathrm{H}$ II-region-like spaxels seen in the line ratio and metallicity. $\mathrm{H} \alpha$ imaging by Dopita et al. (2002) shows further knots of star formation along the spiral arms, and our nuclear spectra also show signs of an aging stellar population in the nucleus of IC 4734.

IRAS F19115-2124 (ESO 593-IG008). Väisänen et al. (2008b) analyze the morphology of this close pair in detail using adaptive optics $K$-band images and suggest the possibility of a triple-galaxy interaction. Kinematic modeling can be satisfied by an interacting pair, however, and indicates that though this system is classified as a "close pair," it is actually just entering first pericenter (J. Barnes 2015, private communication). The metallicity gradient in this system, however, has already flattened considerably (Rich et al. 2012).

IRAS F20551-4250 (ESO 286-IG019). This late-stage merger shows ambiguous AGN signatures. The Chandra X-ray data show extended soft emission inconsistent with an AGN (Ptak et al. 2003; Grimes et al. 2005; Iwasawa et al. 2009, 2011), but Franceschini et al. (2003) classify the galaxy as an AGN based on the XMM-Newton data. There is no $[\mathrm{Ne}$ v] detection in the mid-IR, though the $6.2 \mu \mathrm{m}$ PAH EW is very low $(0.1 \mu \mathrm{m})$, more consistent with AGN emission (Petric et al. 2011; Stierwalt et al. 2013). Imanishi et al. (2010), Sani et al. (2008), and Nardini et al. (2008) also claim signs of a heavily obscured AGN with IR data. Our IFS data are dominated by a post-starburst spectrum and composite LINER emission, with the emission-line velocity dispersion distribution centered around $100 \mathrm{~km} \mathrm{~s}^{-1}$ and trailing to $300 \mathrm{~km} \mathrm{~s}^{-1}$. The emission-line maps show the lowest emission-line ratios near the nucleus with increasing ratios farther away, more consistent with shock excitation in the optical rather than a low- 
luminosity AGN. The Na D absorption line profile in this galaxy also appears to have broad blue wings consistent with an outflow (e.g., Rupke et al. 2005), though no fit to the complex was performed. Our observations are in agreement with previous optical spectra of the nucleus, classified as H II dominated (Veilleux et al. 1995; Kewley et al. 2001b), coupled with extended shock excitation.

IRAS F21330-3846 (ESO 343-IG013). This closely interacting pair shows tidal tails in the available DSS image. Our IFS data show that both disks are dominated primarily by $\mathrm{H}_{\text {II }}$ emission. The line ratio diagnostic diagrams show a composite component, which corresponds primarily to the outskirts of the northern galaxy. The ionized emission between the two systems also has higher line ratio and velocity dispersion than the disks, consistent with shock excitation possibly caused directly by the interaction, as seen in systems like Stephan's Quintet and the Taffy Galaxies (Cluver et al. 2010; Guillard et al. 2010; Peterson et al. 2012) as opposed to outflow-driven shocks.

IRAS F21453-3511 (NGC 7130). NGC 7130 is an isolated face-on spiral galaxy with a previously observed Seyfert 2 nucleus (Phillips et al. 1983). The AGN dominates the mid-IR spectra in the nucleus and is detected in X-ray as well (Iwasawa et al. 2011; Petric et al. 2011). Because it is nearby and nearly uninclined, our IFS spectroscopy resolves well the AGN and starburst component in this galaxy, showing spiral arms dominated by star formation and a strong Seyfert component in the nucleus. The distribution of spaxels in the diagnostic diagram and the line ratio maps show this well and agree with Monreal-Ibero et al. (2010), who also observed this system. $\mathrm{Na} \mathrm{D}$ absorption shows a blueshifted component that may be due to an AGN-driven outflow. Further analysis of the WiFeS data for NGC 7130 is presented in Leslie et al. (2014).

IRAS F22467-4906 (ESO 239-IG002). The nuclear regions of this compact late-stage merger have very high $[\mathrm{N} \mathrm{III} / \mathrm{H} \alpha$ and $[\mathrm{S}$ II] $/ \mathrm{H} \alpha$ line ratios, with moderate $[\mathrm{O}$ III] $/ \mathrm{H} \beta$ ratios indicating composite/LINER behavior. The nucleus also shows a poststarburst spectrum in the blue, with older stellar features in the tidal debris to the southeast of the nucleus. There is some detection of low-level $\mathrm{H}$ II-region-like emission in the tidal arm to the SE as well, likely similar to the star formation in IRAS F10038-3338, likely related to the shock-induced star formation in NGC 7252 described by Chien \& Barnes (2010). The Chandra data show a point-like source detected in the hard band, while the soft X-rays extend to the $\mathrm{N}$ and $\mathrm{S}$ and the overall hardness of the X-ray data is not consistent with an AGN (Iwasawa et al. 2011). There is a very broad blueshifted Na D absorption feature indicating an outflow that may be driving shocks in the galaxy. The velocity dispersion distribution seems consistent with low-velocity shocks with some highvelocity components, but the limited spatial resolution of our data in this system makes this more difficult to discern.

IRAS F23128-5919 (ESO 148-IG002). The nuclei in this closely interacting ULIRG are separated by a projected distance of about $4 \mathrm{kpc}$ and show distinctly different properties in our spectra. The northern nucleus has line ratio and velocity dispersion values consistent with star formation. The southern nucleus is about three times brighter at $24 \mu \mathrm{m}$ and hosts an Xray- and IR-detected AGN (Franceschini et al. 2003; Iwasawa et al. 2011; Petric et al. 2011), which are mirrored in the enhanced composite/Seyfert line ratios and broadened velocity dispersions seen in our IFS diagnostic diagrams and emission- line maps. The emission-line components associated with the composite/Seyfert emission show broad velocity dispersions of $200-400 \mathrm{~km} \mathrm{~s}^{-1}$, though there is still a significant contribution below $100 \mathrm{~km} \mathrm{~s}^{-1}$, likely owing to the ongoing star formation in both nuclei. The broad component shows signs of rotation, while the underlying narrow component seems to show none, indicating that most of the narrow emission may be coming from the non-AGN galaxy. We do not detect a blueshifted component in fits to the Na D component. Further analysis of the WiFeS data for ESO 148-IG002 is presented in Leslie et al. (2014).

\section{REFERENCES}

Alonso-Herrero, A., Rieke, G. H., Rieke, M. J., \& Scoville, N. Z. 2002, AJ, 124,166

Annibali, F., Bressan, A., Rampazzo, R., et al. 2010, A\&A, 519, 40 Armus, L., Charmandaris, V., Bernard-Salas, J., et al. 2007, ApJ, 656, 148

Armus, L., Heckman, T. M., \& Miley, G. K. 1989, ApJ, 347, 727

Armus, L., Heckman, T. M., \& Miley, G. K. 1990, ApJ, 364, 471

Armus, L., Mazzarella, J. M., Evans, A. S., et al. 2009, PASP, 121, 559

Arribas, S., \& Colina, L. 2003, ApJ, 591, 791

Arribas, S., Colina, L., Bellocchi, E., Maiolino, R., \& Villar-Martín, M. 2014, A\&A, 568, A14

Arribas, S., Colina, L., Monreal-Ibero, A., et al. 2008, A\&A, 479, 687

Baldwin, J. A., Phillips, M. M., \& Terlevich, R. 1981, PASP, 93, 5

Belfiore, F., Maiolino, R., Bundy, K., et al. 2015, MNRAS, 449, 867

Bessell, M. S. 1999, PASP, 111, 1426

Blain, A. W., Smail, I., Ivison, R. J., Kneib, J.-P., \& Frayer, D. T. 2002, PhR, 369,111

Blanc, G. A., Heiderman, A., Gebhardt, K., Evans, N. J., II, \& Adams, J. 2009, ApJ, 704, 842

Bundy, K., Bershady, M. A., Law, D. R., et al. 2015, ApJ, 798, 7

Bundy, K., Fukugita, M., Ellis, R. S., et al. 2009, ApJ, 697, 1369

Chien, L.-H., \& Barnes, J. E. 2010, MNRAS, 407, 43

Cluver, M. E., Appleton, P. N., Boulanger, F., et al. 2010, ApJ, 710, 248

Croom, S. M., Lawrence, J. S., Bland-Hawthorn, J., et al. 2012, MNRAS, 421,872

Darling, J., \& Giovanelli, R. 2002, AJ, 124, 100

Dasyra, K. M., Yan, L., Helou, G., et al. 2008, ApJ, 680, 232

Davies, R. L., Rich, J. A., Kewley, L. J., \& Dopita, M. A. 2014, MNRAS, 439, 3835

de Ravel, L., Le Fèvre, O., Tresse, L., et al. 2009, A\&A, 498, 379

Desai, V., Armus, L., Spoon, H. W. W., et al. 2007, ApJ, 669, 810

Dib, S., Bell, E., \& Burkert, A. 2006, ApJ, 638, 797

Dopita, M., Hart, J., McGregor, P., et al. 2007, Ap\&SS, 310, 255

Dopita, M., Rhee, J., Farage, C., et al. 2010, Ap\&SS, 95

Dopita, M. A., Ho, I.-T., Dressel, L. L., et al. 2015, ApJ, 801, 42

Dopita, M. A., Pereira, M., Kewley, L. J., \& Capaccioli, M. 2002, ApJS, 143,47

Efstathiou, G. 2000, MNRAS, 317, 697

Elmegreen, D. M., \& Elmegreen, B. G. 2006, ApJ, 651, 676

Epinat, B., Amram, P., Balkowski, C., \& Marcelin, M. 2010, MNRAS, 401, 2113

Eracleous, M., Hwang, J. A., \& Flohic, H. M. L. G. 2010, ApJ, 711, 796

Farage, C. L., McGregor, P. J., Dopita, M. A., \& Bicknell, G. V. 2010, ApJ, 724,267

Farrah, D., Bernard-Salas, J., Spoon, H. W. W., et al. 2007, ApJ, 667, 149

Ferland, G. J., \& Netzer, H. 1983, ApJ, 264, 105

Fernandes, I. F., de Carvalho, R., Contini, T., \& Gal, R. R. 2004, MNRAS, 355,728

Fosbury, R. A. E., Mebold, U., Goss, W. M., \& Dopita, M. A. 1978, MNRAS, 183,549

Franceschini, A., Braito, V., Persic, M., et al. 2003, MNRAS, 343, 1181

Fu, H., Yan, L., Scoville, N. Z., et al. 2010, ApJ, 722, 653

Genzel, R., Burkert, A., Bouchè, N., et al. 2008, ApJ, 687, 59

Genzel, R., Förster Schreiber, N. M., Rosario, D., et al. 2014, ApJ, 796, 7

Genzel, R., Lutz, D., Sturm, E., et al. 1998, ApJ, 498, 579

González Delgado, R. M., Cerviño, M., Martins, L. P., Leitherer, C., \& Hauschildt, P. H. 2005, MNRAS, 357, 945

Green, A. W., Glazebrook, K., McGregor, P. J., et al. 2010, Natur, 467, 684

Grimes, J. P., Heckman, T., Strickland, D., \& Ptak, A. 2005, ApJ, 628, 187

Guillard, P., Boulanger, F., Cluver, M. E., et al. 2010, A\&A, 518, A59

Haan, S., Surace, J. A., Armus, L., et al. 2011, AJ, 141, 100 
Halpern, J. P., \& Steiner, J. E. 1983, ApJL, 269, L37

Henkel, C., \& Wilson, T. L. 1990, A\&A, 229, 431

Hinshaw, G., Weiland, J. L., Hill, R. S., et al. 2009, ApJS, 180, 225

Ho, I.-T., Kewley, L. J., Dopita, M. A., et al. 2014, MNRAS, 444, 3894

Ho, L. C. 1999, AdSpR, 23, 813

Ho, L. C. 2009, ApJ, 699, 626

Howell, J. H., Armus, L., Mazzarella, J. M., et al. 2010, ApJ, 715, 572

Imanishi, M., Nakagawa, T., Shirahata, M., Ohyama, Y., \& Onaka, T. 2010, ApJ, 721, 1233

Inami, H., Armus, L., Charmandaris, V., et al. 2013, ApJ, 777, 156

Ishida, C. M. 2004, PhD thesis, Univ. Hawaii

Iwasawa, K., Sanders, D. B., Evans, A. S., et al. 2009, ApJL, 695, L103

Iwasawa, K., Sanders, D. B., Teng, S. H., et al. 2011, A\&A, 529, A106

Joye, W. A., \& Mandel, E. 2003, in ASP Conf. Ser. 295, Astronomical Data Analysis Software and Systems XII, ed. H. E. Payne, R. I. Jedrzejewski \& R. N. Hook (San Francisco, CA: ASP), 489

Kauffmann, G., Heckman, T. M., Tremonti, C., et al. 2003, MNRAS, 346, 1055

Kewley, L. J., Dopita, M. A., Sutherland, R. S., Heisler, C. A., \& Trevena, J. 2001a, ApJ, 556, 121

Kewley, L. J., Groves, B., Kauffmann, G., \& Heckman, T. 2006, MNRAS, 372,961

Kewley, L. J., Heisler, C. A., Dopita, M. A., \& Lumsden, S. 2001b, ApJS, 132,37

Kewley, L. J., Rupke, D., Jabran Zahid, H., Geller, M. J., \& Barton, E. J. 2010, ApJL, 721, L48

Kim, D.-C., Sanders, D. B., Veilleux, S., Mazzarella, J. M., \& Soifer, B. T. 1995, ApJS, 98, 129

Kovo, O., \& Contini, T. 1999, in IAU Symp. 193, Wolf-Rayet Phenomena in Massive Stars and Starburst Galaxies, ed. K. A. van der Hucht, G. Koenigsberger \& P. R. J. Eenens (San Francisco, CA: ASP), 604

Leslie, S., Rich, J., Kewley, L., \& Dopita, M. 2014, MNRAS, 444, 1842

Lutz, D., Veilleux, S., \& Genzel, R. 1999, ApJL, 517, L13

Markwardt, C. B. 2009, in ASP Conf. Ser. 411, Astronomical Data Analysis Software and Systems XVIII, ed. D. A. Bohlender, D. Durand \& P. Dowler (San Francisco, CA: ASP), 251

Martin, J. M., Bottinelli, L., Dennefeld, M., et al. 1988, A\&A, 195, 71

Mazzarella, J. M., Iwasawa, K., Vavilkin, T., et al. 2012, AJ, 144, 125

Medling, A. M., U, V., Rich, J. A., et al. 2015, MNRAS, 448, 2301

Monaco, P. 2004, MNRAS, 352, 181

Monreal-Ibero, A., Arribas, S., \& Colina, L. 2006, ApJ, 637, 138

Monreal-Ibero, A., Arribas, S., Colina, L., et al. 2010, A\&A, 517, A28

Moustakas, J., \& Kennicutt, R. C., Jr. 2006, ApJS, 164, 81

Murphy, T. W., Jr., Armus, L., Matthews, K., et al. 1996, AJ, 111, 1025

Nardini, E., Risaliti, G., Salvati, M., et al. 2008, MNRAS, 385, L130

Osterbrock, D. E. 1989, Astrophysics of Gaseous Nebulae and Active Galactic Nuclei (Mill Valley, CA: Univ. Science Books)

Pereira-Santaella, M., Diamond-Stanic, A. M., Alonso-Herrero, A., \& Rieke, G. H. 2010, ApJ, 725, 2270

Pérez-Montero, E., \& Contini, T. 2009, MNRAS, 398, 949
Peterson, B. W., Appleton, P. N., Helou, G., et al. 2012, ApJ, 751, 11

Petric, A. O., Armus, L., Howell, J., et al. 2011, ApJ, 730, 28

Phillips, M. M., Charles, P. A., \& Baldwin, J. A. 1983, ApJ, 266, 485

Poggianti, B. M., \& Wu, H. 2000, ApJ, 529, 157

Ptak, A., Heckman, T., Levenson, N. A., Weaver, K., \& Strickland, D. 2003, ApJ, 592, 782

Pych, W. 2004, PASP, 116, 148

Rich, J. A., Dopita, M. A., Kewley, L. J., \& Rupke, D. S. N. 2010, ApJ, 721, 505

Rich, J. A., Kewley, L. J., \& Dopita, M. A. 2011, ApJ, 734, 87

Rich, J. A., Kewley, L. J., \& Dopita, M. A. 2014, ApJL, 781, L12

Rich, J. A., Torrey, P., Kewley, L. J., Dopita, M. A., \& Rupke, D. S. N. 2012, ApJ, 753, 5

Rigopoulou, D., Spoon, H. W. W., Genzel, R., et al. 1999, AJ, 118, 2625

Rothberg, B., \& Fischer, J. 2010, ApJ, 712, 318

Rupke, D. S., Veilleux, S., \& Sanders, D. B. 2005, ApJS, 160, 115

Rupke, D. S. N., Kewley, L. J., \& Chien, L. 2010, ApJ, 723, 1255

Rush, B., Malkan, M. A., Fink, H. H., \& Voges, W. 1996, ApJ, 471, 190

Sánchez, S. F., Kennicutt, R. C., Gil de Paz, A., et al. 2012, A\&A, 538, A8

Sanders, D. B., Mazzarella, J. M., Kim, D., Surace, J. A., \& Soifer, B. T. 2003, AJ, 126, 1607

Sanders, D. B., Soifer, B. T., Elias, J. H., et al. 1988, ApJ, 325, 74

Sani, E., Risaliti, G., Salvati, M., et al. 2008, ApJ, 675, 96

Sarzi, M., Shields, J. C., Schawinski, K., et al. 2010, MNRAS, 402, 2187

Scoville, N. Z., Evans, A. S., Thompson, R., et al. 2000, AJ, 119, 991

Sharp, R. G., \& Bland-Hawthorn, J. 2010, ApJ, 711, 818

Silk, J. 2001, MNRAS, 324, 313

Soto, K. T., Martin, C. L., Prescott, M. K. M., \& Armus, L. 2012, ApJ, 757, 86

Spoon, H. W. W., Tielens, A. G. G. M., Armus, L., et al. 2006, ApJ, 638, 759

Staveley-Smith, L., Norris, R. P., Chapman, J. M., et al. 1992, MNRAS, 258,725

Stierwalt, S., Armus, L., Surace, J. A., et al. 2013, ApJS, 206, 1

Sturm, E., Lutz, D., Tran, D., et al. 2000, A\&A, 358, 481

Thompson, T. A., Quataert, E., \& Murray, N. 2005, ApJ, 630, 167

U, V., Medling, A., Sanders, D., et al. 2013, ApJ, 775, 115

Väisänen, P., Mattila, S., Kniazev, A., et al. 2008b, MNRAS, 384, 886

Väisänen, P., Ryder, S., Mattila, S., \& Kotilainen, J. 2008a, ApJL, 689, L37

Veilleux, S., Kim, D., Sanders, D. B., Mazzarella, J. M., \& Soifer, B. T. 1995 , ApJS, 98, 171

Veilleux, S., \& Osterbrock, D. E. 1987, ApJS, 63, 295

Veilleux, S., \& Rupke, D. S. 2002, ApJL, 565, L63

Westmoquette, M. S., Clements, D. L., Bendo, G. J., \& Khan, S. A. 2012, MNRAS, 424, 416

Westmoquette, M. S., Smith, L. J., \& Gallagher, J. S., III 2011, MNRAS, 414, 3719

Wild, V., Rosales-Ortega, F., Falcón-Barroso, J., et al. 2014, A\&A, 567, A132

Wu, Y., Charmandaris, V., Huang, J., Spinoglio, L., \& Tommasin, S. 2009, ApJ, 701, 658

Yuan, T., Kewley, L. J., \& Sanders, D. B. 2010, ApJ, 709, 884

Zahid, H. J., Kewley, L. J., \& Bresolin, F. 2011, ApJ, 730, 137 